VERSION: August 13, 2021

Preprint typeset using LTEX style emulateapj v. 12/16/11

\title{
PROBING THE COSMIC X-RAY AND MEV GAMMA-RAY BACKGROUND RADIATION THROUGH THE ANISOTROPY
}

\author{
Yoshiyuki Inoue $^{1}$, Kohta Murase $^{2}$, Grzegorz M. MAdeJski $^{1}, \&$ YASUnobu Uchiyama $^{1,3}$ \\ ${ }^{1}$ Kavli Institute for Particle Astrophysics and Cosmology, Department of Physics, Stanford University and SLAC National Accelerator Laboratory, 2575 Sand \\ Hill Road, Menlo Park, CA 94025, USA \\ ${ }^{2}$ Hubble Fellow, School of Natural Sciences, Institute for Advanced Study, 1 Einstein Dr. Princeton NJ 08540 and \\ ${ }^{3}$ Department of Physics, Rikkyo University, 3-34-1 Nishi-Ikebukuro, Toshima-ku, Tokyo, Japan 171-8501 \\ Version: August 13, 2021
}

\begin{abstract}
While the cosmic soft X-ray background is very likely to originate from individual Seyfert galaxies, the origin of the cosmic hard X-ray and $\mathrm{MeV}$ gamma-ray background is not fully understood. It is expected that Seyferts including Compton thick population may explain the cosmic hard X-ray background. At MeV energy range, Seyferts having non-thermal electrons in coronae above accretion disks or MeV blazars may explain the background radiation. We propose that future measurements of the angular power spectra of anisotropy of the cosmic X-ray and $\mathrm{MeV}$ gamma-ray backgrounds will be key to deciphering these backgrounds and the evolution of active galactic nuclei (AGNs). As AGNs trace the cosmic large-scale structure, spatial clustering of AGNs exists. We show that $e$-ROSITA will clearly detect the correlation signal of unresolved Seyferts at $0.5-2 \mathrm{keV}$ and $2-10 \mathrm{keV}$ bands and will be able to measure the bias parameter of AGNs at both bands. Once the future hard X-ray all sky satellites achieve the sensitivity better than $10^{-12} \mathrm{erg} / \mathrm{cm}^{2} / \mathrm{s}$ at $10-30 \mathrm{keV}$ or $30-50 \mathrm{keV}$ - although this is beyond the sensitivities of current hard X-ray all sky monitors - angular power spectra will allow us to independently investigate the fraction of Compton-thick AGNs in all Seyferts. We also find that the expected angular power spectra of Seyferts and blazars in the $\mathrm{MeV}$ range are different by about an order of magnitude, where the Poisson term, so-called shot noise, is dominant. Current and future $\mathrm{MeV}$ instruments will clearly disentangle the origin of the $\mathrm{MeV}$ gamma-ray background through the angular power spectrum.

Subject headings: cosmology: diffuse radiation - galaxies: active - X-rays: diffuse background - gamma rays : theory
\end{abstract}

\section{INTRODUCTION}

The cosmic X-ray background (CXB) is an isotropic, apparently diffuse X-ray emission in the Universe which was discovered about 50 years ago (Giacconi et al. 1962). It is often assumed that the CXB has been conclusively shown to be the integrated light produced via the accretion process of active galactic nuclei (AGNs), in particular Seyferts, hosting supermassive black holes. This might be correct below $\sim 5 \mathrm{keV}$. Emission from active galaxies has indeed been resolved by the deep X-ray surveys by Chandra in the broad $0.5-2 \mathrm{keV}$ and 2 $10 \mathrm{keV}$ bands. Those objects account for $80-90 \%$ of the CXB (Mushotzky et al. 2000; Giacconi et al. 2002; Alexander et al. 2003a.b; Bauer et al. 2004). However, energy-resolved studies indicate that the resolved fraction of the CXB decreases with energy as $80-90 \%$ over $2-8 \mathrm{keV}, \sim 60 \%$ over $6-8 \mathrm{keV}$, and $\sim 50 \%$ beyond $8 \mathrm{keV}$ (Worsley et al. 2004, 2005).

Above $\sim 2 \mathrm{keV}$, the CXB cannot be due to superposition of unabsorbed AGNs, mainly type I Seyferts. Those objects show a typical continuum photon index of $\Gamma=1.9$ below $10 \mathrm{keV}$ (Nandra \& Pounds 1994; Reeves \& Turner 2000; Piconcelli et al. 2005), different from that of CXB $\Gamma=1.4$ at 2-8 keV (De Luca \& Molendi 2004). Instead, this unresolved, hard component is generally attributed to the emission from absorbed Seyferts, the so-called type II Seyferts, which might be buried in dusty tori. A superposition of such sources with varying degrees of photoelectric absorption by the circumnuclear material can cause the total spectrum to appear harder than spectra of unabsorbed Seyferts, but this re-

yinoue@slac.stanford.edu quires some fine-tuning of absorption properties of sources as a function of redshift and luminosity. Various population synthesis models successfully explain the CXB by introducing appropriate number of absorbed Seyferts (see e.g. Ueda et al. 2003; Treister \& Urrv 2005; Gilli et al. 2007). However, recent studies (Treister et al. 2009) showed that the number of Compton thick AGNs, which are a class of absorbed Seyferts and whose column density is larger than the inverse of the Thomson cross section, is a factor of 3-4 less than that expected in the population synthesis models at least locally (see also Ajello et al. 2012a). This may pose a serious problem to our current knowledge of the origin of the CXB.

By contrast, the origin of the cosmic $\mathrm{MeV}$ gamma-ray background at $\sim 1-10 \mathrm{MeV}$ has been an intriguing mystery. The Seyfert spectra adopted in population synthesis models of the CXB cannot explain this component because of the assumed exponential cutoff at a few hundred $\mathrm{keV}$, where thermal hot corona above the accretion disk is assumed. Above $100 \mathrm{MeV}$, it is known that superposition of blazars (e.g. Padovani et al. 1993; Inoue \& Totani 2009; Abdo et al. 2010b; Aiello et al. 2012b), starburst galaxies (e.g. Soltan \& Juchniewicz 1999; Ackermann et al. 2012b), and radio galaxies (e.g. Padovani et al. 1993; Inoue 2011; Di Mauro et al. 2013) explains most of the total background flux. These populations may contribute to the MeV background as well. However, the background spectrum from several hundreds $\mathrm{keV}$ to several tens $\mathrm{MeV}$ is smoothly connected to the CXB spectrum and is much softer (photon index $\Gamma \sim 2.8$ ) than the $\mathrm{GeV}$ component (Fukada et al. 1975; Watanabe et al. 1997; Weidenspointner et al. 2000), in- 
dicating a different origin from that above $100 \mathrm{MeV}$ (e.g. Sreekumar et al. 1998; Abdo et al. 2010a).

A few candidates have been proposed to explain the $\mathrm{MeV}$ background. One was the nuclear-decay gammarays from Type Ia supernovae (SNe Ia; Clayton \& Ward 1975; Zdziarski 1996; Watanabe et al. 1999). However, on the basis of the latest measurements of the cosmic SN Ia rates, recent studies show that the $\mathrm{MeV}$ background flux expected from $\mathrm{SNe}$ Ia is about an order of magnitude lower than observed (Ahn et al. 2005; Strigari et al. 2005; Horiuchi \& Beacom 2010). Seyferts can naturally explain the $\mathrm{MeV}$ background including the smooth connection to the CXB (Schoenfelder 1978; Field \& Rogers 1993; Inoue et al. 2008). Comptonized photons produced by non-thermal electrons in hot coronae surrounding accretion disks can produce the $\mathrm{MeV}$ power-law tail (Inoue et al. 2008). Such non-thermal electrons are expected to exist if the corona is heated by magnetic reconnection (Liu et al. 2002). There is also a class of blazars, called MeV blazars, whose spectra peak at MeV energies (Blom et al. 1995; Sambruna et al. 2006). These MeV blazars could potentially contribute to the MeV background as well (Ajello et al. 2009). Radio galaxies have been also discussed as the origin of the MeV background (Strong et al. 1976). However, recent studies show that the expected background flux from radio galaxies is $\sim 10 \%$ of the total $\mathrm{MeV}$ background flux (Massaro \& Ajello 2011; Inoue 2011). Annihilation of the dark matter particles has also been discussed (Olive \& Silk 1985; Ahn \& Komatsu 2005a b; Ando \& Komatsu 2006; Rasera et al. 2006; Lawson \& Zhitnitsky 2008), but those are less natural dark matter candidates, with a mass scale of $\mathrm{MeV}$ energies, rather than $\mathrm{GeV}-\mathrm{TeV}$ dark matter candidates. In either case, there is little observational evidence of $\mathrm{MeV}$ emission from these candidates and a quantitative estimate is not easy due to the sensitivity of the $\mathrm{MeV}$ measurements.

The angular power spectrum of the background radiation will shed new light on these problems, since it reflects the distribution of its origin in the entire sky. The angular power spectrum is obtained by performing a spherical harmonics transformation of the sky intensity map after subtracting foregrounds and point sources. As an aside, both theoretical and observational studies of the cosmic microwave background (CMB) anisotropy has allowed us to precisely determine the total content in the Universe (e.g. Komatsu et al. 2009, 2011). In the gammaray sky, the anisotropy is becoming key to understanding the origin of the GeV background (Ando \& Komatsu 2006; Ando et al. 2007a b; Ackermann et al. 2012a; Cuoco et al. 2012; Harding \& Abazaiian 2012; Ando \& Komatsu 2013).

The anisotropy in the X-ray band has been well studied with tools such as auto-correlation functions (e.g. de Zotti et al. 1990; Carrera et al. 1993; Chen et al. 1994; Soltan \& Hasinger 1994; Sołtan et al. 1999; Scharf et al. 2000; Sołtan et al. 2001; Sliwa et al. 2001; Kushino et al. 2002) and cross-correlations with galaxies, clusters, and CMB (e.g. Lahav et al. 1993; Mivaii et al. 1994; Carrera et al. 1995; Barcons et al. 1995; Roche et al. 1995; Soltan et al. 1996; Trever \& Lahav 1996; Soltan et al. 1997; Newsam et al. 1999; Stevenson et al. 2002; Boughn \& Crittenden 2004a, 2005). Theoretically, analytical formalism has been developed to calculate the angular power spectra of the cosmic background radiation in X-ray and gamma-ray band (e.g. Gao et al. 1990; Lahav et al. 1997; Barcons et al. 1998;
Ando \& Komatsu 2006; Ando et al. 2007a b). However, angular power spectra of the cosmic background radiation from Seyferts and blazars have not been studied in the context of the latest X-ray luminosity function (XLF) in the $\mathrm{X}$-ray and $\mathrm{MeV}$ gamma-ray range, while those from SNe Ia (Zhang \& Beacom 2004) and MeV dark matter (Ando \& Komatsu 2006) have been discussed extensively.

Galaxies and AGNs are hosted by dark matter halos, but they trace the dark matter distribution with some bias. This bias factor is a key to understanding the formation mechanism, environment, and evolution of AGNs, since it represents the clustering strength of a source population compared with dark matter. The bias parameter determined from various AGN surveys is controversial. While the correlation functions of the X-ray local AGNs detected by the ROentgen SATellite (ROSAT) suggest the value close to unity (Vikhlinin \& Forman 1995; Mullis et al. 2004), those from Chandra and X-ray Multi-Mirror Mission - Newton (XMM-Newton) suggest stronger clustering (Yang et al. 2003; Basilakos et al. 2005; Gandhi et al. 2006). Moreover, the bias parameter inferred from X-ray AGNs is higher at $0<z<3$ that from optically selected quasars (Koutoulidis et al. 2013).

Angular power spectrum allows us to study the bias factor of AGNs from another aspect, since the correlation term of angular power spectrum depends on the bias parameter. The Ginga satellite has studied the angular structure of the CXB down to 0.2 degrees at $4-12 \mathrm{keV}$ in the regions of the North Galactic Pole and the North Ecliptic Pole (Carrera et al. 1993). Since no significant deviation from isotropy is found, the bias parameter of AGNs cannot be constrained. The previous analysis of the High Energy Astronomy Observatory $(H E A O) 1 \mathrm{~A}-2 \mathrm{X}$-ray sky map with a XLF of Seyferts indicated the bias parameter close to unity (Boughn \& Crittenden 2004b), whereas Scharf et al. (2000) showed the angular power spectrum of the HEAO1 A-2 X-ray sky map is dominated by the shot noise which is independent of the bias parameter. Revnivtsev et al. (2008) reported CXB intensity variation up to $\sim 2 \%$ on angular scales of $20-40$ degrees with the Rossi X-ray Timing Explorer (RXTE). However, fluctuation at the smaller angular scales or the detection of the correlation term was not reported.

Here, we evaluate the angular power spectra in the soft X-ray region, the hard X-ray region, and the $\mathrm{MeV}$ region with the latest Seyfert and blazar XLFs. The new era of the X-ray and $\mathrm{MeV}$ gamma-ray Universe is nearing, with current and future missions such as Astro$H$ (Takahashi et al. 20121, Nuclear Spectroscopic telescope array (NuStar; Harrison et al. 2013), extended ROentgen Survey with an Imaging Telescope Array (e-Rosita; Merloni et al. 2012]3, CAST (Nakazawa et al. 2012), DUAL (von Ballmoos et al. 2012), GRIPS (Greiner et al. 2012)4, and SMILE (Takada et al. 2011) 5. Here, we discuss the detectability of the anisotropy at each energy region by these future missions and future possible studies through the anisotropy.

This paper is organized as follows. In Section 2, we present the spectral model of Seyferts and blazars. In Section 3, we describe the XLF of Seyferts and blazars. In Section 4, we

\footnotetext{
1 http://astro-h.isas.jaxa.jp

2 http://www.nustar.caltech.edu

3 http: / / www . mpe.mpg . de/eROSITA

4 http://www.grips-mission.eu

5 http: //www-cr.scphys.kyoto-u.ac.jp/research/MeV-gamma/en/
} 
briefly review the formulations to calculate the angular power spectra of X-ray and MeV background anisotropy. Results of the angular power spectra are shown in Section 5. Discussions and conclusions are given in Section 6. Throughout this paper, we adopt the standard cosmological parameters of $\left(h, \Omega_{M}, \Omega_{\Lambda}\right)=(0.7,0.3,0.7)$.

\section{X-RAY AND MEV GAMMA-RAY EMISSION FROM ACTIVE} GALACTIC NUCLEI

\subsection{Seyferts}

The X-ray spectra of Seyferts represent a superposition of multiple physical processes in the galactic nucleus and surrounding gas. Phenomenologically the components of these spectra are measured to show a power-law continuum with a cutoff at $\sim 300 \mathrm{keV}$ in the form of $E^{-\Gamma} \exp \left(-E / E_{c}\right)$, absorption from surrounding gas, emission lines, and a continuum hump, the called "reflection component", and a soft excess of emission at $\leq 2 \mathrm{keV}$, often approximated by a blackbody or a power-law. According to the currently popular unification models, this primary continuum may be absorbed by circumnuclear material, with the degree of absorption related to the inclination of the symmetry axis of the accretion disk: low-luminosity variants of such absorbed AGN are Seyfert II galaxies.

Physically the primary continuum is thought to originate from multiple Compton scatterings of thermal disk photons in an optically thin (or at most moderately thick) hot corona above the disk (see e.g. Katz 1976; Pozdniakov et al. 1977; Sunyaev \& Titarchuk 1980) with the high energy cutoff which roughly represents the temperature of the corona (see e.g. Zdziarski et al. 1994). The continuum slope (photon index) is determined by the Compton $y$-parameter which is a combination of the coronal temperature and optical depth. Reflection component appears as a result of the Compton reprocessed emission and bound-free absorption of the primary continuum by cold matter in the accretion disk and the surrounding gas (Lightman \& White 1988; Magdziarz \& Zdziarski 1995).

As an aside, it is worth mentioning that X-ray binaries (XRBs) are also accretion disk systems, although the central black hole mass is of solar mass size. In fact, X-ray spectra of Seyferts resemble those of XRBs in hard state (Zdziarski et al. 1999). XRBs can extend this emission to MeV region with a power-law (McConnell et al. 1994; Gierliński et al. 1999). Although $\mathrm{MeV}$ power-law tail has never been confirmed in Seyferts, some models predict the existence of such $\mathrm{MeV}$ power-law tail (see e.g. Inoue et al. 2008), in which thermal and non-thermal electrons coexist in the corona above the accretion disk. This scenario is naturally expected if the hot corona is heated by the magnetic reconnection (Liu et al. 2002). Non-thermal electrons are known to exist at Solar flares (e.g. Shibata et al. 1995) and Earth's magnetotail (Lin et al. 2005) where magnetic reconnection occurs. In the context of this model, with non-thermal component having $4 \%$ of the total electron energy, $\mathrm{MeV}$ gamma-ray background can be explained by the same population of Seyferts that makes up the CXB as shown below (Inoue et al. 2008). Observationally, the Oriented Scintillation Spectroscopy Experiment (OSSE) clearly detected emission up to $500 \mathrm{keV}$ in the spectrum of the brightest Seyfert 1 NGC 4151 (Johnson et al. 1997). Beyond $\sim 200 \mathrm{keV}$, the spectrum steepens. By combining the flux upper limit data above $500 \mathrm{keV}$, the maximum allowed non-thermal fraction is 15\% (Johnson et al. 1997).

In this paper, we consider two intrinsic spectral models for Seyferts. One is thermal spectral model which has a power- law continuum with a cutoff (see e.g. Ueda et al. 2003). We adopt $\Gamma=1.9$ and $E_{c}=300 \mathrm{keV}$. The other is thermal plus non-thermal spectral model (see Inoue et al. 2008, for details). We adopt the same parameters as in Inoue et al. (2008), but setting the thermal cutoff-energy at $300 \mathrm{keV}$. For the Compton reflection component, we use a Compton reflection model (Magdziarz \& Zdziarski 1995) (developed for the XSPEC package as "pexrav"), assuming a solid angle of $2 \pi$, an inclination angle of $\cos i=0.5$, and solar abundance for all elements. To calculate absorbed spectra, we use an absorption model called "wabs" developed for the XSPEC package.

\subsection{Blazars}

The multi-wavelength studies of blazars show that the overall spectra have two pronounced continuum components: one peaking between infrared and X-rays and another in the gamma-ray regime (Fossati et al. 1998; Kubo et al. 1998). The lower energy component is produced by synchrotron radiation, while the higher energy component is produced by the inverse Compton (IC) scattering of ambient seed photons by the same electrons (see e.g. Ulrich et al.1997; Ghisellini et al. 1998). The target seed photon can be synchrotron radiation in the jet, in the synchrotron self-Compton (SSC) model (see e.g. Jones et al. 1974), or external radiation such as emission from accretion disk, broad line region, or dusty torus, in the external radiation Compton (ERC) model (see e.g. Dermer \& Schlickeiser 1993; Sikora et al. 1994).

Blazars can be classified into three subclasses by their spectra: high-energy peaked BL Lacertae objects (HBLs), lowenergy peaked BL Lac objects (LBLs), and flat spectrum radio quasars (FSRQs). The overall emission of HBLs may be explained by the SSC scenario, while that of FSRQs may be explained by the ERC scenario. X-ray spectra of HBLs, lowluminosity blazars, show the softest spectra among them with photon index $\Gamma \sim 2-3$, and this X-ray emission is the highest observable energy tail of the synchrotron component. Since FSRQs significantly contribute to the cosmic X-ray and MeV gamma-ray background as compared to BL Lacs (Ajello et al. 2009), we focus on FSRQs only for blazars hereinafter. X-ray spectra of FSRQs are harder and this emission is the lowest observable energy tail of the IC component. For FSRQs, we assume the blazar spectral energy distribution (SED) with an empirical double power-law model:

$$
\frac{d N}{d E} \propto\left[\left(\frac{E}{E_{b}}\right)^{\Gamma_{1}}+\left(\frac{E}{E_{b}}\right)^{\Gamma_{2}}\right]^{-1},
$$

where we set $E_{b}=3 \mathrm{MeV}, \Gamma_{1}=1.6$, and $\Gamma_{2}=2.9$ following Ajello et al. (2009). The average photon index of FSRQs observed by Swift-BAT at $15-55 \mathrm{keV}$ is $1.6 \pm 0.3$ (Ajello et al. 2009). Theoretically, FSRQs' spectra are expected to show a break and spectral softening at MeV band (see e.g. Inoue \& Takahara 1996). However, $E_{b}$ and $\Gamma_{2}$ of FSRQs at MeV band are not constrained by observations due to the difficulty of the MeV gamma-ray measurement. The values of them here are artificially selected to explain the $\mathrm{MeV}$ background by FSRQs. If $E_{b}$ is at $\sim \mathrm{MeV}$ region, FSRQs can significantly contribute to the $\mathrm{MeV}$ background by choosing appropriate $\Gamma_{2}$. At GeV band, Fermi has observed 310 FSRQs whose mean value of photon index above $0.1 \mathrm{GeV}$ is $2.42 \pm 0.17$ (Ackermann et al. 2011). However, the photon index of the MeV gamma-ray background spectrum is $\sim 2.8$ (Watanabe et al. 1997). This suggests $\Gamma_{2} \sim 2.8$ to explain the 
$\mathrm{MeV}$ background by FSRQs. Therefore, if the MeV background is explained by FSRQs, MeV and GeV FSRQs may be different populations or FSRQs have a complex SED shape.

\section{X-RAY LUMINOSITY FUNCTION}

To obtain the background radiation spectrum and angular power spectrum in the X-ray band, an XLF of sources is required. XLF gives the comoving number density at each luminosity and each redshift. We briefly review XLFs of Seyferts and blazars in this section.

\subsection{Seyferts}

Various X-ray surveys allowed to determine the evolution of Seyferts including unobscured and moderately obscured sources (see e.g. Ueda et al. 2003; Hasinger et al. 2005; Gilli et al. 2007; Aird et al.2010). X-ray photons above $\sim 2 \mathrm{keV}$ are relatively unaffected by absorption for moderate column density $\left(N_{\mathrm{H}} \lesssim 10^{23} \mathrm{~cm}^{-2}\right)$. XLF studies at the $2-10 \mathrm{keV}$ band have revealed that luminosity-dependent density evolution (LDDE) models reproduce the observed XLFs at various redshift and luminosity ranges (Ueda et al. 2003; La Franca et al. 2005; Silverman et al. 2008; Ebrero et al. 2009; Yencho et al. 2009). LDDE predicts that the shape of the XLF changes with redshift, with the faint-end slope flattening as redshift increases. This evolution is also characterized by a shift in the peak of the space density towards lower redshifts for lower luminosities, so-called downsizing. Aird et al. (2010) suggested a more complex evolution model, luminosity and density evolution (LADE) model. LADE predicts a fixed shape of the XLF at all redshifts, but varies the normalization of the XLF.

In this study, we follow the Ueda et al. (2003) LDDE $\mathrm{XLF}$ at $2-10 \mathrm{keV}$, since LDDE is confirmed to be adequate at 0.5-2 keV (e.g. Mivaii et al. 2000; Hasinger et al. 2005) and 2-10 keV (e.g. Ueda et al. 2003; La Franca et al. 2005; Silverman et al. 2008; Ebrero et al. 2009; Yencho et al. 2009) and the distribution function of the neutral hydrogen column density is not available for the LADE model (Aird et al. 2010). The comoving number density $\rho_{X}$ in the LDDE is given as:

$$
\rho_{X}\left(L_{X}, z, N_{\mathrm{H}}\right)=\rho_{X}\left(L_{X}, 0\right) f\left(L_{X}, z\right) \eta\left(N_{\mathrm{H}} ; L_{X}, z\right),
$$

where $L_{X}$ is the X-ray luminosity, $z$ is the redshift, and $N_{\mathrm{H}}$ is the neutral hydrogen column density. $\rho_{X}\left(L_{X}, 0\right)$ is the AGN $\mathrm{XLF}$ at present. This is characterized by the faint-end slope index $\gamma_{1}$, the bright-end slope index $\gamma_{2}$, and the break luminosity $L_{X}^{*}$, as:

$$
\rho_{X}\left(L_{X}, 0\right)=A_{X}\left[\left(\frac{L_{X}}{L_{X}^{*}}\right)^{\gamma_{1}}+\left(\frac{L_{X}}{L_{X}^{*}}\right)^{\gamma_{2}}\right]^{-1},
$$

where $A_{X}$ is the normalization parameter having a dimension of volume ${ }^{-1}$.

The function $f\left(L_{X}, z\right)$ describes the density evolution, which is given by the following form:

$$
f\left(L_{X}, z\right)= \begin{cases}(1+z)^{p_{1}} & z \leq z_{c}\left(L_{X}\right), \\ \left(1+z_{c}\left(L_{X}\right)\right)^{p_{1}}\left(\frac{1+z}{1+z_{c}\left(L_{X}\right)}\right)^{p_{2}} z>z_{c}\left(L_{X}\right),\end{cases}
$$

where $z_{c}$ is the redshift of evolutionary peak, given as

$$
z_{c}\left(L_{X}\right)=\left\{\begin{array}{lr}
z_{c}^{*} & L_{X} \geq L_{a}, \\
z_{c}^{*}\left(L_{X} / L_{a}\right)^{\alpha} L_{X}<L_{a} .
\end{array}\right.
$$

The function $\eta\left(N_{\mathrm{H}} ; L_{\mathrm{X}}, z\right)$ describes the distribution of absorption column density, which is given by the following form in the XLF (Ueda et al. 2003):

$$
\eta\left(N_{\mathrm{H}} ; L_{\mathrm{X}}, z\right)= \begin{cases}2-\frac{5+2 \epsilon}{1+\epsilon} \psi\left(L_{\mathrm{X}}, z\right) & \left(20.0 \leq \log N_{\mathrm{H}}<20.5\right), \\ \frac{1}{1+\epsilon} \psi\left(L_{\mathrm{X}}, z\right) & \left(20.5 \leq \log N_{\mathrm{H}}<23.0\right),(6) \\ \frac{\epsilon}{1+\epsilon} \psi\left(L_{\mathrm{X}}, z\right) & \left(23.0 \leq \log N_{\mathrm{H}}<24.0\right),\end{cases}
$$

where $\epsilon=1.7$ and

$$
\left.\psi\left(L_{X}, z\right)=\min \left\{\psi_{\max }, \max \left[0.47-0.1\left(\log L_{X}-44.0\right), 0\right]\right)\right\},
$$

for which

$$
\psi_{\max }=\frac{1+\epsilon}{3+\epsilon} .
$$

The parameters obtained by the fit to the observed data of $\mathrm{X}$-ray AGNs in Ueda et al. (2003) are shown in Table1. We set the minimum of the X-ray luminosity as $L_{X, \min }=10^{41.5} \mathrm{erg}$ $\mathrm{s}^{-1}$, the same as in Ueda et al. (2003).

As discussed in the introduction, an absorbed Seyfert population can contribute to the CXB significantly at $\gtrsim 10 \mathrm{keV}$. One of the main interests of X-ray AGN studies is the population of the Compton thick AGNs. The column density of the Compton thick AGNs is larger than $N_{\mathrm{H}}=1 / \sigma_{T} \simeq$ $1.5 \times 10^{24} \mathrm{~cm}^{-2}$, where $\sigma_{T}$ is the Thomson scattering cross section. Here, we assume the fraction of the Compton thick AGNs between $24.0 \leq \log N_{\mathrm{H}}<25.0$ to be the same as that of the population at $23.0 \leq \log N_{\mathrm{H}}<24.0$ following Ueda et al. (2003) to explain the CXB by Seyferts. However, recent study of the Swift-BAT hard X-ray AGN samples revealed that the number density of the Compton thick AGNs is a factor of 3-4 less, at least locally, than that required to explain the CXB at hard X-ray (see e.g. Treister et al. 2009). The Compton thick AGN population has not been fully resolved due to the necessity for imaging with hard X-ray instruments, and those, provided with, $N U S T A R$ and Astro- $H$ will resolve this population further beyond the local Universe. As shown below, the angular power spectrum at hard X-rays will be another probe to study the Compton thick AGN population.

Although the distribution function of absorption column density $\eta\left(N_{\mathrm{H}} ; L_{\mathrm{X}}, z\right)$ is not available for the LADE model (Aird et al. 2010), we can test the LADE model by assuming the same absorption column density distribution as in the LDDE. When we adopt the LADE model (Aird et al. 2010), the overall normalization of the CXB needs to decrease by a factor of $\sim 30 \%$ and no change is required to the spectral shape. Regarding the angular power spectrum, its shape does not change but the normalization will decrease by a factor of $\sim 50 \%$ and $\sim 10 \%$ at $0.5-2 \mathrm{keV}$ and at $2-10 \mathrm{keV}$, respectively, by assuming the $e$-Rosita all sky survey sensitivity (see Figures. (2) and 3). Our conclusion, which depends on the shape of the angular power spectrum, will not significantly change even if we adopt the LADE model for the Seyfert evolution.

\subsection{Blazars}

Gamma-ray studies of blazars indicated that LDDE well represents the evolution of EGRET blazars (Narumoto \& Totani 2006; Inoue \& Totani 2009). The recent study of Fermi FSRQs confirmed that LDDE provides a good description of the evolution of FSRQs Ajello et al. 2012b). However, in the X-ray band, the situation is different. Due to the beaming effect, the number density of blazars is less than Seyferts. Currently, Swift-BAT has done the deepest survey above $15 \mathrm{keV}$ (Baumgartner et al. 2012). Ajello et al. 
TABLE 1

THE PARAMETERS OF THE AGN XLF

\begin{tabular}{ccr}
\hline \hline & $\begin{array}{c}\text { Ueda et al. 2003 } \\
\text { Seyfert } \\
2-10 \mathrm{keV}\end{array}$ & $\begin{array}{c}\text { Ajello et al. 2009 } \\
\text { FSRQ } \\
15-55 \mathrm{keV}\end{array}$ \\
\hline$A_{X}{ }^{a}$ & $50.4 \pm 3.3$ & $0.533 \pm 0.104$ \\
$\log _{10} L_{X}^{* b}$ & $43.94_{-0.21}^{+0.26}$ & 44.0 \\
$\gamma_{1}$ & $0.86 \pm 0.15$ & - \\
$\gamma_{2}$ & $2.23 \pm 0.13$ & $3.45 \pm 0.20$ \\
$z_{c}^{*}$ & 1.9 & - \\
$\log _{10} L_{a}^{b}$ & 44.6 & - \\
$\alpha$ & $0.335 \pm 0.07$ & - \\
$p_{1}$ & $4.23 \pm 0.39$ & $3.72 \pm 0.50$ \\
$p_{2}$ & -1.5 & - \\
$p_{3}$ & - & $-0.32 \pm 0.08$
\end{tabular}

${ }^{a}$ In units of $10^{-7} \mathrm{Mpc}^{-3}$

${ }^{\mathrm{b}}$ In units of $\mathrm{erg} / \mathrm{s}$.

(2009) studied the cosmological evolution of X-ray blazars using three years of data from Swift-BAT AGN survey with 26 FSRQs and 12 BL Lacs. The evolution of the FSRQs is reproduced by a PLE model. Since the number of samples for the Ajello et al. (2009) XLF is limited, in the future study, it is necessary to use the XLF converted from other wavelength LFs such as radio or gamma-ray (e.g. Aiello et al. 2012a), and by using the luminosity correlation (see Inoue 2011, for the case of radio galaxies). In this paper, we adopt this latest blazar XLF model (Ajello et al. 2009).

The blazar XLF by Aiello et al. (2009) is given in the form of pure luminosity evolution (PLE) model. PLE predicts the same shape of XLF at all redshifts, but shifts the shape with luminosity. The comoving number densities $\rho_{X}$ in the PLE is given as:

$$
\rho_{X}\left(L_{X}, z\right)=\rho_{X}\left(L_{X} / e(z), 0\right) .
$$

The local XLF is characterized by a single power-law function with the slope index $\gamma_{2}$ and the break luminosity $L_{X}^{*}$, as:

$$
\rho_{X}\left(L_{X}, 0\right)=A_{X}\left(\frac{L_{X}}{L_{X}^{*}}\right)^{-\gamma_{2}} .
$$

The function $e(z)$ describes the evolution factor independent of luminosity, which is given by the following forms:

$$
e(z)=(1+z)^{p_{1}+p_{3} z}
$$

The parameters obtained by the fit to the observed data of Xray FSRQs in A09 are shown in Table 1. They also assume an evolving minimum luminosity as

$$
L_{X, \operatorname{Min}}(z)=L_{X, \operatorname{Min}, 0} \times e(z),
$$

where $L_{\mathrm{X}, \mathrm{Min}, 0}$ is the minimum luminosity as at $z=0$. We set $L_{\mathrm{X}, \mathrm{Min}, 0}=3 \times 10^{44} \mathrm{erg} \mathrm{s}^{-1}$ (Ajello et al. 2009).

\subsection{Cosmic X-ray and MeV Gamma-Ray Background Intensity}

The unresolved background flux at the observed energy $E$ can be expressed as

$I(E)=\int_{0}^{z_{\max }} d z \frac{d^{2} V}{d z d \Omega} \int_{L_{\min }}^{L\left(F_{\lim }(E), z\right)} d L \int_{N_{\mathrm{H}, \text { min }}}^{N_{\mathrm{H}, \max }} d N_{\mathrm{H}} F(L, z, E) \rho\left(L, z, N_{\mathrm{H}}\right.$

where $F(L, z, E)$ is the observed photon flux from a source having a luminosity $L$ at redshift $z$ and $F_{\text {lim }}(E)$ is the flux limit at the energy $E$. In the case of blazars, we ignore the term

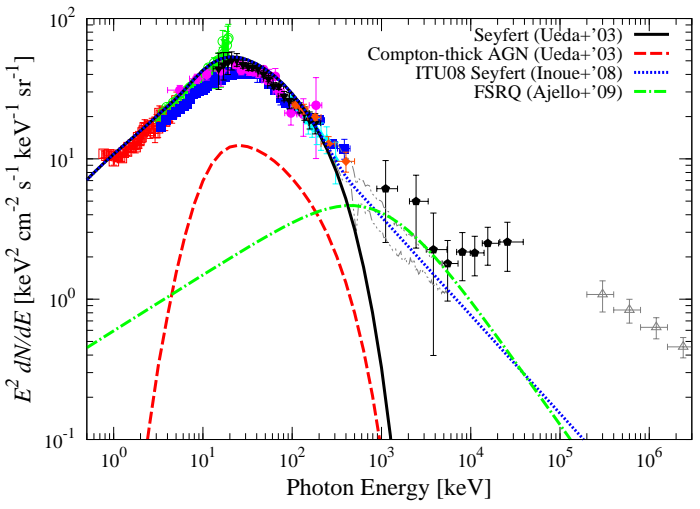

FIG. 1. - The cosmic X-ray and MeV gamma-ray background spectrum. Solid, dashed, dotted, and dot-dashed curve shows the contribution from Seyferts with $20<\log N_{\mathrm{H}}<25$ (Ueda et al. 2003), Compton thick AGNs, i.e. Seyferts with $24<\log N_{\mathrm{H}}<25$ (Ueda et al. 2003), Seyferts with non-thermal electrons in the coronae (Inoue et al. 2008), and FSRQs (Ajello et al. 2009). The cosmic X-ray background spectrum data of ASCA (open squares, Gendreau et al. 1995), RXTE (open circles, Revnivtsev et al. 2005), HEAO-1 A2 (filled squares, Gruber et al. 1999), INTEGRAL (filled circles Churazov et al. 2007), HEAO-1 A4 (filled up-triangles, Kinzer et al. 1997), Swift-BAT (filled down-triangles, Ajello et al. 2008), SMM (triple dot-dashed, Watanabe et al. 1997), NagoyaBallon (filled diamonds, Fukada et al. 1975), COMPTEL (filled hexagons, Weidenspointner et al. 2000), and Fermi (open triangles, Abdo et al. 2010a) are shown in the figure. For the SMM data, the triple dot-dashed curve shows the 1- $\sigma$ uncertainty region. Although Fermi has measured the background spectrum up to $100 \mathrm{GeV}$ (Abdo et al. 2010a), we plot their result up to 30 $\mathrm{GeV}$ to show the CXB and the MeV background clearly.

of $N_{\mathrm{H}}$ in Eq. 13. When we consider the total (resolved + unresolved) background flux, we substitute $L\left(F_{\lim }(E), z\right)$ to $L_{\max }$, where we set $L_{\max }=10^{48} \mathrm{erg} / \mathrm{s}$ for Seyferts (Ueda et al. 2003) and $L_{\max }=10^{50} \mathrm{erg} / \mathrm{s}$ for blazars (Ajello et al. 2009).

Fig. 1 1 shows the contribution to the cosmic X-ray and MeV gamma-ray background spectra from Seyferts (Ueda et al. 2003), Compton-thick AGNs (Ueda et al. 2003), Seyferts with non-thermal tails (Inoue et al. 2008), and FSRQs (Ajello et al. 2009) using the spectral models in Section 2 and the LFs in Section 3 together with observational data of ASCA (Gendreau et al. 1995), RXTE (Revnivtsev et al. 2005), HEAO-1 A2 (Gruber et al. 1999), INTEGRAL (Churazov et al. 2007), HEAO-1 A4 (Kinzer et al. 1997), Swift-BAT (Ajello et al. 2008), SMM (Watanabe et al. 1997), Nagoya-Ballon (Fukada et al. 1975), COMPTEL (Weidenspointner et al. 2000), and Fermi (Abdo et al. 2010a). By including the Compton-thick AGNs, we can adequately fit the CXB spectrum at $1-200 \mathrm{keV}$ by the Seyfert population (Ueda et al. 2003). Seyferts with nonthermal electrons in coronae (Inoue et al. 2008) and FSRQs (Ajello et al. 2009) can explain the $\mathrm{MeV}$ background.

\section{COSMIC X-RAY AND MEV GAMMA-RAY BACKGROUND ANISOTROPY}

In this section, we review the formalism to analytically calculate angular power spectra of cosmic background anisotropy (Peebles 1980; Lahav et al. 1997; Ando \& Komatsu 2006). Detailed formulation is given in Appendix $\mathrm{A}$

), The angular power spectrum of the CXB from point sources

${ }^{6}$ Although the background flux literally means the unresolved flux from the sky, the cosmic infrared, optical, and X-ray background flux usually mean the total flux which includes flux from resolved sources and unresolved sources (e.g. Inoue et al. 2013; Ueda et al. 2003; ; Aiello et al. 2009). 
such as AGNs is given by

$$
C_{l}=C_{l}^{P}+C_{l}^{C},
$$

where the first term $C_{l}^{P}$ is the Poisson (shot noise) term and the second term $C_{l}^{C}$ is the correlation term (Peebles 1980; Ando et al. 2007a b). The shot noise term does not depend on the multipole $l$, while the correlation term reflects the intrinsic spatial correlation of sources. The multipole term $l$ is related to the angular separation $\theta$ in the sky as $l \simeq 180 / \theta$, where $\theta$ is the angular scale in the sky in units of degrees.

The two terms are related to the spatial power spectrum through

$$
\begin{aligned}
C_{l}^{P}= & \int d z \frac{d^{2} V}{d z d \Omega} \int d L \int d N_{\mathrm{H}} F(L, z)^{2} \rho_{X}\left(L, z, N_{\mathrm{H}}\right) \\
C_{l}^{C}= & \int d z \frac{d^{2} V}{d z d \Omega} P_{\operatorname{lin}}\left(k=\frac{l}{r(z)}, z\right) \\
& \times\left[\int d L \int d N_{\mathrm{H}} b_{\mathrm{AGN}}(L, z) F(L, z) \rho_{X}\left(L, z, N_{\mathrm{H}}\right)\right]^{2},
\end{aligned}
$$

where $P_{\text {lin }}(k, z)$ is the power spectrum of the linear matter density fluctuation as a function of the wave number $k=l / r$, $r(z)$ is the proper distance, and $b_{\mathrm{AGN}}(L, z)$ is the bias factor of AGNs against dark matter. We use the linear transfer function given in Eisenstein \& $\mathrm{Hu}$ (1999) for $P_{\text {lin }}(k, z)$. The integration range is the same as Eq. 13 for the unresolved background flux. We also assume the Limber approximation which means that fluctuation does not change strongly and which is valid for small angular separation, $l \gtrsim 6$ corresponding to $\theta \lesssim 30^{\circ}$. In the case of blazars, we ignore the term of $N_{\mathrm{H}}$ in Eqs. 15 and 16 .

The 1- $\sigma$ statistical error in the measurement of the angular power spectrum is given by

$$
\left(\delta C_{l}\right)^{2}=\frac{2 C_{l}^{2}}{(2 l+1) \Delta l f_{\text {sky }}},
$$

where $\Delta l$ is the bin size in the multipole space and $f_{\text {sky }}$ is a fraction of the sky covered by observations (see Ando et al. $2007 \mathrm{a}, \mathrm{b}$, for details). Hereinafter we assume the all sky survey, with $f_{\text {sky }}=1$ (such as $e$-Rosita) and set $\Delta l=0.5 l$. Eq. 17 shows that the statistical error is reduced by removing as many point sources as possible or by measuring as high $l(\operatorname{small} \theta)$ as possible.

\subsection{Bias Factor of Active Galactic Nuclei}

The bias factor of AGNs is a key to understanding the environment of the AGN formation in the cosmic history. The bias factor represents the clustering strength of AGNs compared with dark matter (See Eq. A19). Clustering of AGNs has been studied with large samples in the optical large survey, such as the Two-degree Field Quasar Redshift Survey (Croom et al. 2005; Porciani \& Norberg 2006) and the Sloan Digital Sky Survey (SDSS Li et al. 2006; Ross et al. 2009; Shen et al. 2009). The bias evolves from $b_{\mathrm{AGN}} \sim 1.4$ at $z=0.5$ (Ross et al. 2009), $b_{\mathrm{AGN}} \sim 3$ at $z=2.2$ (Ross et al. 2009), to $b_{\mathrm{AGN}} \sim 10$ at $z=4.0$ (Shen et al. 2009). In X-rays, many papers have explored the angular clustering of AGNs (Vikhlinin \& Forman 1995; Akvlas et al. 2000; Yang et al. 2003; Basilakos et al. 2004; Mullis et al. 2004; Gandhi et al. 2006; Puccetti et al. 2006; Carrera et al. 2007; Mivaii et al. 2007; Plionis et al. 2008; Ebrero et al. 2009; Bradshaw et al.
2011; Elyiv et al. 2012; Koutoulidis et al. 2013). However, the bias parameter of AGNs has not been determined to agree neither between optical and X-ray nor amongst various X-ray studies. While both the angular and 3D correlation function of the X-ray bright AGNs detected by the ROSAT suggested that close to unity with the median redshift $z=0.4$ (Vikhlinin \& Forman 1995; Mullis et al. 2004), those from Chandra and XMM-Newton suggested stronger clustering (Yang et al. 2003; Basilakos et al. 2005; Gandhi et al. 2006). The inferred bias parameter from XMM-Newton Large Scale Structure survey (Gandhi et al. 2006) is $\sim 3.7$ at the median redshift $z=0.7$ (Ando et al. 2007b). The most recent Xray study based on $1466 \mathrm{X}$-ray AGN samples at $0<z<3$ suggested $b_{\mathrm{AGN}}=2.26$ at the redshift $z=0.976$ (Koutoulidis et al. 2013). They also showed that the bias of X-ray AGNs is significantly higher than those of optically selected AGNs at each redshift. In this study, although it is known that $b_{\mathrm{AGN}}>1$ (Koutoulidis et al. 2013), we conservatively set $b_{\mathrm{AGN}}=1$ independent of for redshift and luminosity for the simplicity, unless noted otherwise.

\section{RESULTS}

\section{1. $0.5-2 \mathrm{keV}$ and 2-10 keV band}

Each panel of fig. 2 shows the results for the angular power spectra of Seyferts at $0.5-2 \mathrm{keV}$ for different sensitivity limit with $1-\sigma$ error bars. We adopt $b_{\mathrm{AGN}}=1$ and $f_{\text {sky }}=1$ here. We find that it would be possible to measure the correlation term of Seyferts with the sensitivity of $10^{-13} \mathrm{erg} / \mathrm{cm}^{2} / \mathrm{s}$ or better. At the large multipole region $l \gtrsim 500$ (corresponding to $\sim 22$ arcmin), the deviation of total angular power spectrum from the poisson term is hardly seen. As fainter point sources are resolved, the Poisson term is reduced and the correlation term will be more clearly detected.

The angular power spectra of Seyferts at $2-10 \mathrm{keV}$ are shown in Fig. 3 for various sensitivity limits. We can measure the correlation term of Seyferts with the sensitivity of $10^{-12} \mathrm{erg} / \mathrm{cm}^{2} / \mathrm{s}$ or better. Similar to the case of $0.5-2 \mathrm{keV}$, at the large multipole region $l \gtrsim 500$, the deviation of total angular power spectrum from the poisson term is hardly seen.

$e$-Rosita will perform an all sky survey with the sensitivity of $10^{-14} \mathrm{erg} / \mathrm{cm}^{2} / \mathrm{s}$ at soft band (corresponding to $0.5-2$ $\mathrm{keV}$ ) and $10^{-13} \mathrm{erg} / \mathrm{cm}^{2} / \mathrm{s}$ at hard band (corresponding to 2$10 \mathrm{keV}$ ) with a 4-year survey (Merloni et al. 2012). The point spread function of $e$-Rosita is $\sim 28$ arcsec (corresponding to $l \sim 2.3 \times 10^{4}$ ) at $1 \mathrm{keV}$ for the survey mode. $e$-Rosita will clearly detect the angular power spectrum of CXB and its correlation signal around $10 \lesssim l \lesssim 1000$ at both of $0.5-2 \mathrm{keV}$ and $2-10 \mathrm{keV}$ even with $b_{\mathrm{AGN}}=1$, if the CXB at these energy bands is composed of Seyferts. Since the Poisson term does not depend on the multipole $l$, we can derive the Poisson term using $C_{l}$ at $l \gtrsim 500$.

Figs. 4 and $\pi$ show the total (Poisson + correlation) angular power spectra from Seyferts at $0.5-2 \mathrm{keV}$ for the sensitivity limit of $10^{-14} \mathrm{erg} / \mathrm{cm}^{2} / \mathrm{s}$ and at $2-10 \mathrm{keV}$ for the sensitivity limit of $10^{-13} \mathrm{erg} / \mathrm{cm}^{2} / \mathrm{s}$, respectively, to demonstrate the capability of $e$-Rosita. Four different bias models are considered. We plot here the cases with constant bias $b_{\mathrm{AGN}}=1$, $b_{\mathrm{AGN}}=3$, the evolving bias parameters inferred from optically selected quasars, and that from X-ray selected AGNs (see the right panel of Fig. 8 of Koutoulidis et al. 2013, for details). At both energy bands, angular power spectra of CXB enable us to clearly distinguish these models by $e$-Rosita

We do not need to divide the angular power spectrum into 


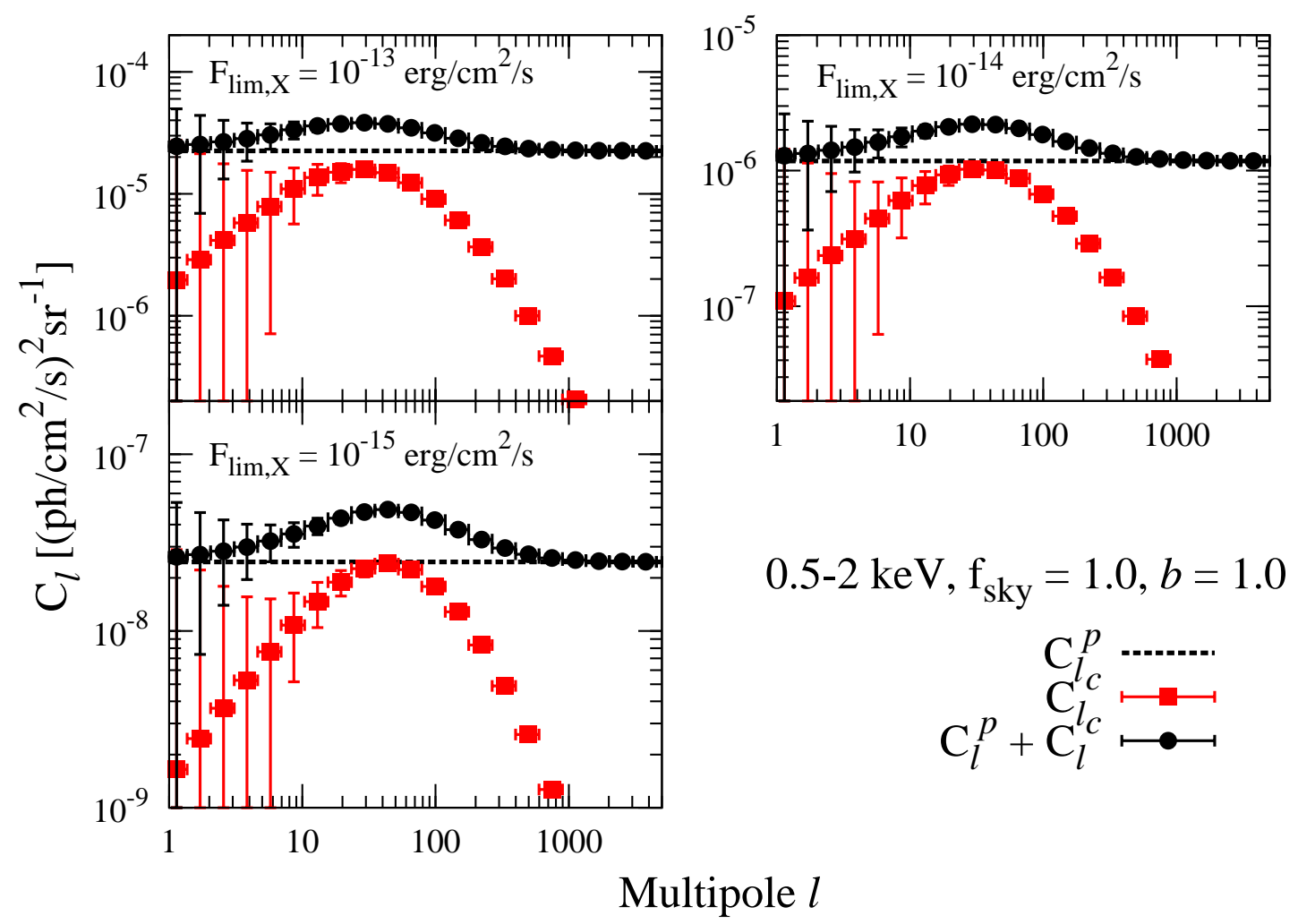

FIG. 2.- Predicted angular power spectra of Seyferts at $0.5-2 \mathrm{keV}$ with $b=1.0$ following the Ueda et al. (2003) XLF. Each panel shows the all sky survey case with the sensitivity limit shown in the panel. Filled circle and filled square points show the total angular power spectrum $\left(C_{l}^{P}+C_{l}^{C}\right)$ and the correlation term $C_{l}^{C}$, respectively. The horizontal dashed line represents the Poisson (Shot noise) term $C_{l}^{P}$. The error bars show the $1 \sigma$ errors with $\Delta l=0.5 l$. The scale of $y$-axis of each panel is different. $0.5-2 \mathrm{keV}$ corresponds the soft band of $e$-Rosita is $0.5-2 \mathrm{keV}$ and its sensitivity limit with a 4-year survey is $F_{\text {lim }} \simeq 10^{-14} \mathrm{erg} / \mathrm{cm}^{2} / \mathrm{s}$ at this band (Merloni et al. 2012).

the Poisson term and the correlation term to derive the bias parameter. The bias will be derived by using the total angular power spectrum which is the directly observable value. Once we obtain the XLF of Seyferts from X-ray deep survey studies (e.g. Ueda et al. 2003; Hasinger et al. 2005; Gilli et al. 2007), the angular power spectrum of CXB obtained by the future $\mathrm{X}$-ray all sky survey will enable us to verify what kind of the bias evolution model is favored. In particular, $e$-Rosita, which covers both energy bands, can evaluate the bias parameter at different energies.

\section{2. $10-30 \mathrm{keV}$ band}

Fig. 6 shows the angular power spectra of Seyferts by all sky survey observations, analogous to Fig. 3 but for 10-30 $\mathrm{keV}$ band. The deviation of the correlation term from the Poisson term is difficult to be measured with the sensitivity limit of $10^{-11} \mathrm{erg} / \mathrm{cm}^{2} / \mathrm{s}$ due to the statistical errors. We need the sensitivity better than $10^{-12} \mathrm{erg} / \mathrm{cm}^{2} / \mathrm{s}$ to detect the correlation term at hard X-ray band. Since the current most sensitive all sky hard X-ray survey is done by Swift-BAT with the sensitivity level of $\sim 10^{-11} \mathrm{erg} / \mathrm{cm}^{2} / \mathrm{s}$ (Baumgartner et al. 2012), one order of magnitude more sensitive instruments are required to measure the correlation term. At $30-50 \mathrm{keV}$, we obtained similar results.

Angular power spectrum at hard X-ray will be another probe to study the Compton thick AGN population. Fig. 7 shows the total (Poisson + correlation) angular power spectra of Seyferts for bias $b_{\mathrm{AGN}}=1$ and 3 and absorption column density with $\log N_{\mathrm{H}}<24.0$ and $\log N_{\mathrm{H}}<25.0$. Each panel represents the case with the sensitivity limit shown in the panel. Low statistical errors are crucial to determine the fraction of the Compton thick AGNs. Hence, point sources should be removed as many as possible to reduce the statistical errors. In the case of $F_{\lim , X}=10^{-11} \mathrm{erg} / \mathrm{cm}^{2} / \mathrm{s}$, it is difficult to distinguish the contribution of Compton thick AGNs with $b_{\mathrm{AGN}}=1$ due to large statistical errors, while it may be possible to see the difference at $l \gtrsim 20$ with $b_{\mathrm{AGN}}=3$. If we can achieve the sensitivity of $F_{\lim , X}=10^{-12} \mathrm{erg} / \mathrm{cm}^{2} / \mathrm{s}$ or better, we can distinguish the contribution of Compton thick AGNs even with $b_{\mathrm{AGN}}=1$.

Can pointing observatories measure angular power spectra of the background radiation? Although the sensitivity limit of hard X-ray all sky survey is still above the required sensitivity for the angular power spectrum study, the pointing observatories such as NuSTAR and Astro- $H$ can achieve the sensitivity of $\sim 10^{-14} \mathrm{erg} / \mathrm{cm}^{2} / \mathrm{s}$ at $10 \mathrm{keV}$ for $100 \mathrm{ks}$ observations (Harrison et al. 2013; Takahashi et al. 2012). As an example, the field of view of NuSTAR is 13 arcmin. If Nustar can do one hundred $100 \mathrm{ks}$ pointing observations in the extragalactic sky during its operation, $f_{\text {sky }}$ will be $10^{-4}$. Following Eq. 17. the statistical error will be two orders of magnitude more enhanced than the case of $f_{\text {sky }}=1$. This large statistical error makes difficult to measure the angular power spectrum of the background radiation with such a small field of view instruments. 


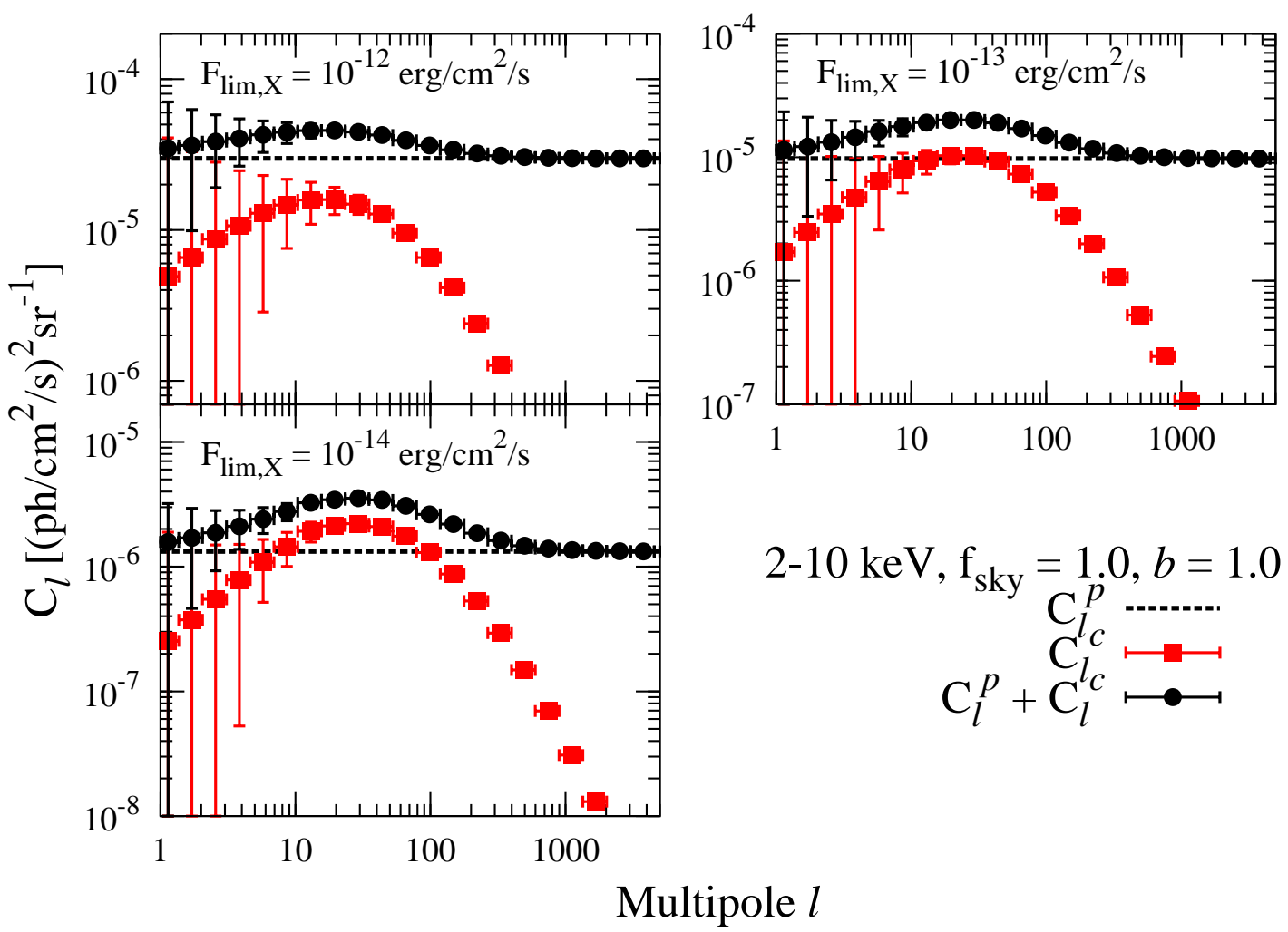

FIG. 3. - Same as Fig. 2 but for $2-10 \mathrm{keV}$ and different sensitivity limits as indicated in each panel. The hard band of $e$-Rosita is $2-10 \mathrm{keV}$ and its sensitivity limit with a 4-year survey is $F_{\mathrm{lim}} \simeq 10^{-13} \mathrm{erg} / \mathrm{cm}^{2} / \mathrm{s}$ at this band (Merloni et al. 2012)

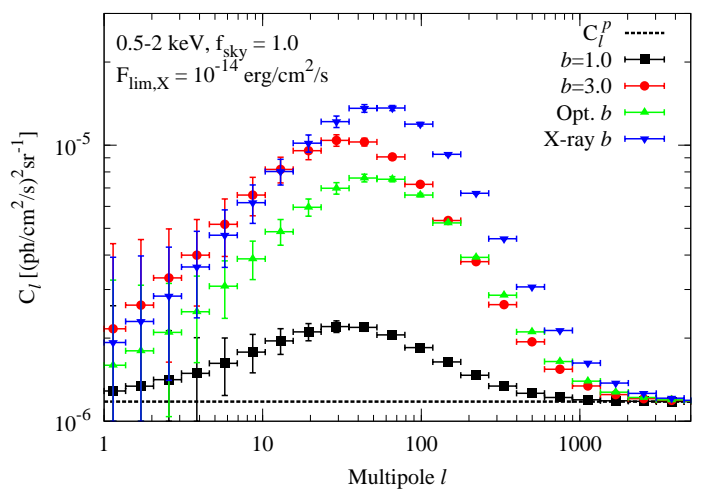

FIG. 4.- Predicted total (Poisson + Correlation) angular power spectra at $0.5-2 \mathrm{keV}$ following the Ueda et al. (2003) XLF. Different bias parameters. We set $F_{\lim , X}=10^{-14} \mathrm{erg} / \mathrm{cm}^{2} / \mathrm{s}$ to demonstrate the capability of $e$ Rosita at $0.5-2 \mathrm{keV}$. All sky survey mode is assumed $f_{\text {sky }}=1$. Square, circle, upper-triangle, and lower-triangle point shows the total angular power spectrum $\left(C_{1}^{P}+C_{1}^{C}\right)$ with $b_{\mathrm{AGN}}=1,3, b_{\mathrm{AGN}}$ following the optical evolution (Koutoulidis et al. 2013), and $b_{\mathrm{AGN}}$ following the X-ray evolution (Koutoulidis et al. 2013) respectively. The horizontal dashed line represents the Poisson (Shot noise) term $C_{l}^{P}$. The error bars show the $1 \sigma$ errors with $\Delta l=0.5 l$.

\subsection{MeV band}

Measurement of MeV gamma-rays is difficult. The dominant process in a detector is Compton scattering and huge background of photons are produced in the $\mathrm{MeV}$ instruments themselves. COMPTEL onboard the CGRO satellite is the only instrument that observed the entire $\mathrm{MeV}$ sky and it discovered only $\sim 30$ gamma-ray sources at $0.75-30 \mathrm{MeV}$

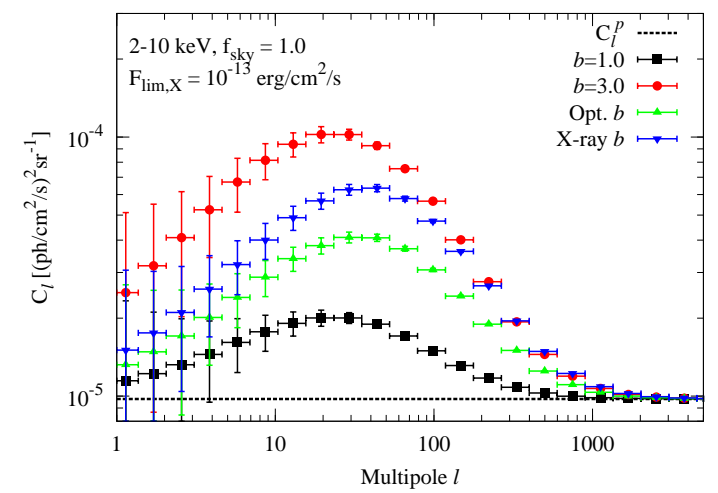

FIG. 5.- Same as Fig. 4 but for 2-10 keV. We set $F_{\lim , X}=10^{-13} \mathrm{erg} / \mathrm{cm}^{2} / \mathrm{s}$ to demonstrate the capability of $e$-Rosita at $2-10 \mathrm{keV}$.

(Schönfelder et al. 2000). Thus, the MeV sky has not been fully investigated. Astro- $H$ which is scheduled to be launched in 2015 will have a sub-MeV instrument, soft gamma-ray detector (SGD; Taiima et al. 2010). The SGD covers a wide energy range from $40 \mathrm{keV}$ up to $600 \mathrm{keV}$ (Takahashi et al. 2012). The field of view (FOV) of the SGD varies with energy. A BGO collimator defines $\sim 10 \mathrm{deg}$ FOV at high energies, while a fine collimator restricts the FOV to $\sim 0.6$ deg below $\sim 150 \mathrm{keV}$. Even though Astro-H is designed to perform pointing observations, the $10 \mathrm{deg}$ FOV of the SGD above $150 \mathrm{keV}$ will allow for a wide sky coverage over the course of the ASTRO-H mission, which is essential to study the $\mathrm{MeV}$ background. A number of future projects are currently proposed to observe the MeV sky such as CAST 


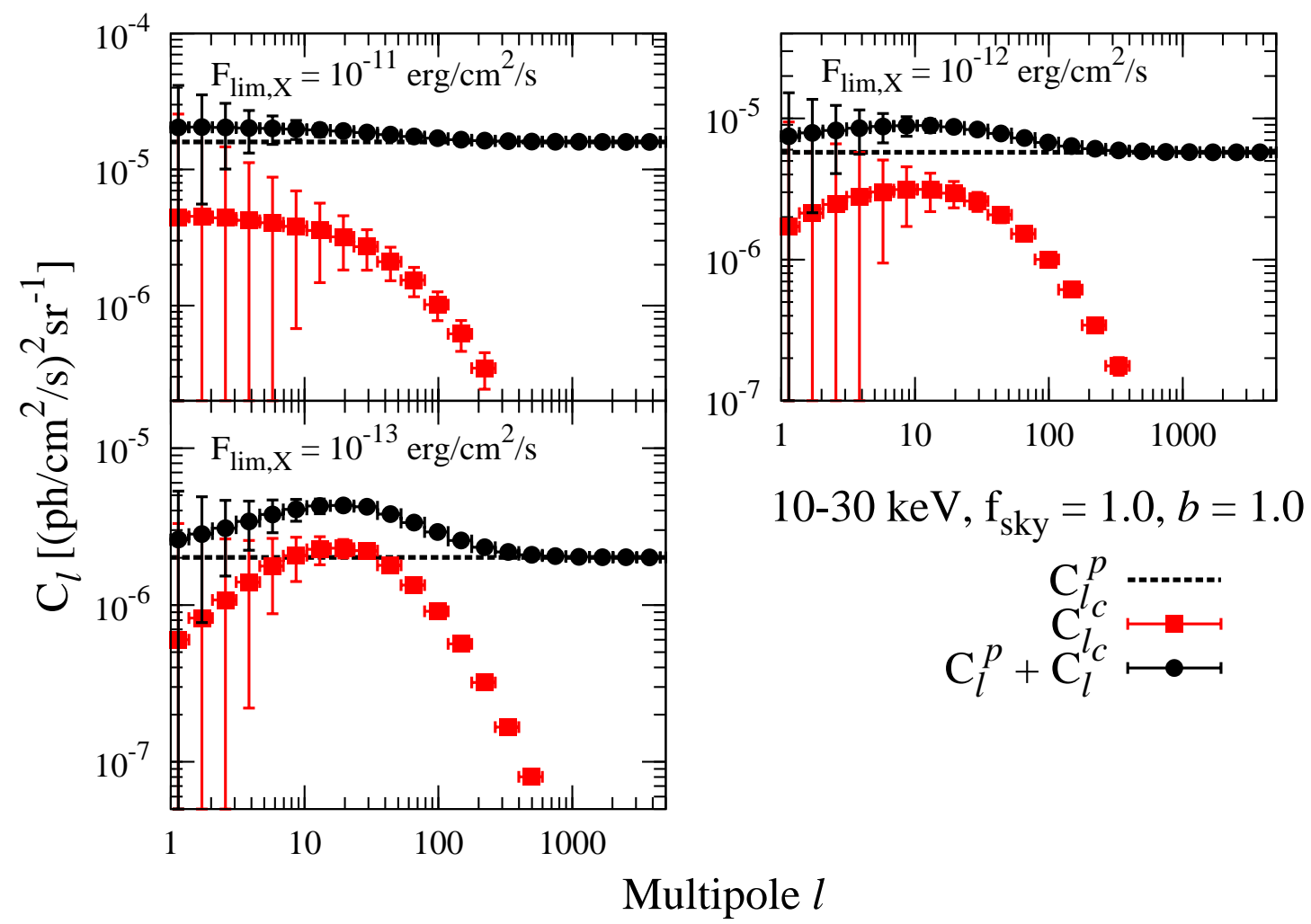

FIG. 6. - Same as Fig. 3 but for $10-30 \mathrm{keV}$.

(Nakazawa et al. 2012), DUAL (von Ballmoos et al. 2012), GRIPS (Greiner et al. 2012) and SMILE (Takada et al. 2011). Recently, various ballon experiments have been carried out to test the performance of instruments (Takada et al. 2011; Bandstra et al. 2011). All of these future instruments will resolve the MeV sky in the coming decades.

Even with those instruments, it is not as easy to resolve the MeV sky as in soft X-ray (see e.g. Bauer et al. 2004). However, one can distinguish the origin of the $\mathrm{MeV}$ background by measuring its angular power spectrum. Fig. 8 shows the Poisson term of the angular power spectra of Seyferts with non-thermal components in coronae (Inoue et al. 2008) and FSRQs (Ajello et al. 2009) with various $\nu F_{\nu}$ sensitivity limit. For reference, we also plot Seyferts with simple thermal cutoff spectra (Ueda et al. 2003), but note that those do not explain the $\mathrm{MeV}$ background. Since the contribution of the correlation term is negligible in this energy region and the assumed flux limits, the angular power spectrum is dominated by the Poisson term. This Poisson term measurement is useful enough to distinguish the origin of the $\mathrm{MeV}$ background. We do not show the expected errors which are highly dependent on the range of observed multipoles. Errors can be estimated from Equation 17. By using high multipole value and wide multipole bin size, the errors will become small. For example, if we select $l=100$ and $\delta l=100(50 \leq l \leq 150)$, the expected uncertainty will be $\delta C_{l} \sim 0.01 C_{l}$.

Left-top panel of Fig. 8 shows the case in which no sources are resolved. We integrate Eq. 15 between $L_{\min }$ and $L_{\max }$. Even if the MeV sky is not resolved into point sources, we can distinguish the origin of the cosmic MeV background. The difference of the $C_{l}^{p}$ of Seyferts (Inoue et al. 2008) and FSRQs (Ajello et al. 2009) is more than an order of magni- tude. The reason why we can clearly distinguish the origin is as follows. Seyferts are fainter but more numerous than blazars. These two differences are able to make future MeV instruments clearly detect the origin of the $\mathrm{MeV}$ gamma-ray sky through the angular power spectrum of the sky (see Eq. 15]. Therefore, ballon experiments may be able to distinguish the origin of the $\mathrm{MeV}$ background sky, although it may suffer from little photon statistics. As Weidenspointner et al. (2000) put an upper limit on the relative deviations from isotropy of the $\mathrm{MeV}$ background, it will be worth revisiting the COMPTEL data in future studies.

In the process of resolving the sources contributing to the $\mathrm{MeV}$ background via improvements of sensitivity and angular resolution, the contribution of Seyferts to the angular power spectrum at sub-MeV region decreases more rapidly than FSRQs. This is because Seyferts dominate the sub-MeV gammaray background at $\lesssim 400 \mathrm{keV}$ (see Fig. 1). With the sensitivity of $10^{-10} \mathrm{erg} / \mathrm{cm}^{2} / \mathrm{s}$ in $\nu F_{\nu}$ close to the sensitivity limit of the COMPTEL (Scharf et al. 2000), we can clearly distinguish the Seyfert scenario (Inoue et al. 2008) vs. the FSRQ scenario (Ajello et al. 2009). Future MeV sky survey instruments will easily distinguish the origin of the $\mathrm{MeV}$ background. However, we note that there will be a very significant background from an instrument itself in the case of the Compton camera technique. Since it may contribute to the angular power spectrum at some level, it is crucial to reduce background events as many as possible. The SGD on board Astro-H is expected to reduce such background significantly (Takahashi et al. 2012).

\section{DISCUSSION AND CONCLUSIONS}

In this paper we have studied the angular power spectra of Seyferts and blazars from $0.5 \mathrm{keV}-10 \mathrm{MeV}$. We have shown 


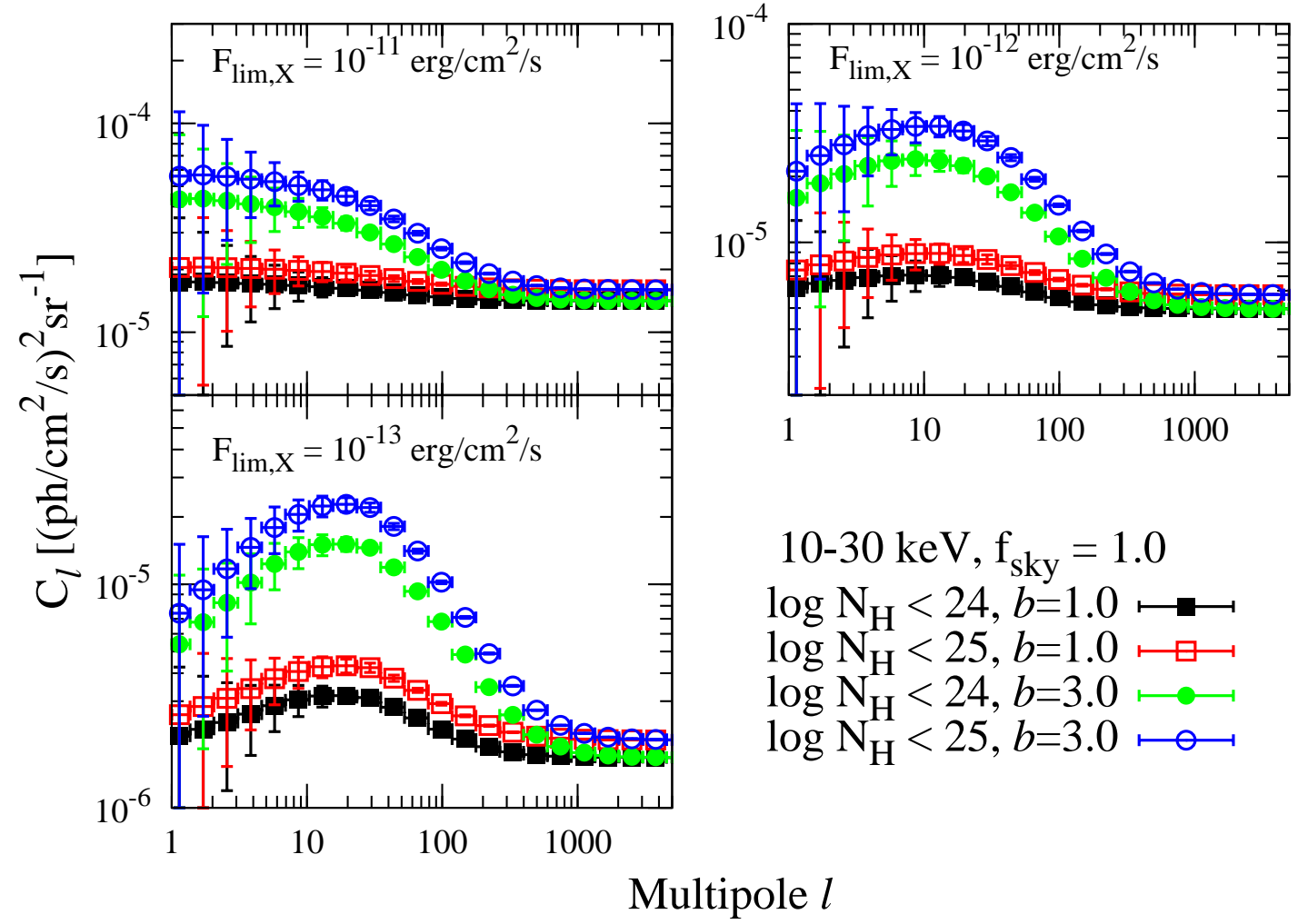

FIG. 7.- Predicted angular power spectra of Seyferts for various $b$ and $\log N_{\mathrm{H}}$ at 10-30 keV following the Ueda et al. (2003) XLF. Each panel shows the all sky survey case $f_{\text {sky }}=1$ with the sensitivity limit shown in the panel. Filled and open points show Seyferts with $\log N_{\mathrm{H}}<24$ and $\log N_{\mathrm{H}}<25$, respectively. Square and circle point shows the case with $b_{\mathrm{AGN}}=1$ and $b_{\mathrm{AGN}}=3$, respectively. The error bars show the $1 \sigma$ errors with $\Delta l=0.5 l$. The scale of $y$-axis of each panel is different.

that $e$-Rosita can detect the spatial clustering of Seyferts including the bias information at $0.5-2 \mathrm{keV}$ and $2-10 \mathrm{keV}$, which is a long standing problem in optical and X-ray AGN survey studies. As the $e$-Rosita AGN sample also allows us to study the bias information (Kolodzig et al. 2013), it will be complementary to each other.

In order to distinguish the population of Compton-thick AGNs, which is believed to be relevant at hard X-ray band (10-30 keV and 30-50 keV), we need to detect the correlation term. However, the sensitivity better than $10^{-12} \mathrm{erg} / \mathrm{cm}^{2} / \mathrm{s}$ is required for this purpose, so the present, best all-sky survey by Swift-BAT (with $\sim 10^{-11} \mathrm{erg} / \mathrm{cm}^{2} / \mathrm{s}$ Baumgartner et al. 2012) is insufficient. Future improvement of the hard X-ray survey instruments is necessary for this study.

At $\mathrm{MeV}$ band, we can clearly distinguish the origin of the $\mathrm{MeV}$ background candidates, Seyferts (Inoue et al. 2008) and FSRQs (Ajello et al. 2009) even with current MeV instruments including ballon experiments. However, this requires that these missions can measure the angular power spectrum of the sky. Future MeV instruments such as SGD onboard Astro- $H$, DUAL, GRIPS, and SMILE will easily disentangle the origin of the MeV background via covering a wide solid angle of the sky with their expected sensitivities.

If the origin of the MeV background is non-thermal emission from Seyfert (Inoue et al. 2008), this implies that magnetic reconnection heats the corona above the disk and accelerate non-thermal electrons in the corona. As discussed in Inoue et al. (2008), this scenario will be also tested by future observations of individual sources. For example, the expected flux from NGC 4151, which is the brightest Seyfert galaxy in the hard X-ray sky (Sazonov et al. 2007), is $\sim 3 \times$ $10^{-5}(E / \mathrm{MeV})^{-0.8} \mathrm{MeV} \mathrm{cm}^{-2} \mathrm{~s}^{-1}$ (Inoue et al. 2008), which can not be detected by COMPTEL but by the future MeV instruments such as CAST, DUAL, GRIPS, and SMILE. If it is FSRQs (Ajello et al. 2009), this implies that there are two distinct FSRQ populations in $\mathrm{MeV}$ and $\mathrm{GeV}$ because of the spectral difference between $\mathrm{MeV}$ and $\mathrm{GeV}$ background. This will suggest that there are two different populations in FSRQs between $\mathrm{MeV}$ and $\mathrm{GeV}$. This may pose a problem to the AGN unification scheme (Urry \& Padovani 1995). Therefore, probing the $\mathrm{MeV}$ background is another handle on to understanding AGN physics.

In our study, we use the power spectrum of linear dark matter fluctuation (Eisenstein \& Hu 1999). At a small angular separation, however, effects of non-linear dark matter fluctuation on the correlation term can not be ignored (Seliak 2000). Non-linear contributions will become important at the scale of $\lesssim 1.5 h^{-1} \mathrm{Mpc}$ (Koutoulidis et al. 2013). This corresponds to $\tilde{l} \gtrsim \sim 600$ at $z \sim 0.3$. $\sim 50 \%$ of the unresolved CXB flux comes from inside of $z=0.3$ at the sensitivity of $e$-Rosita at soft and hard band. Since the correlation term has a peak at $l \sim 100$, the non-linear effect will not change our results significantly.

The required multipole scale for the study of angular power spectrum at X-ray bands is $l \lesssim 500$. This corresponds to $\theta \gtrsim$ 22 arcmin. The point spread function of $e$-Rosita is $\sim 28$ arc $\mathrm{sec}$ at $1 \mathrm{keV}$ for the survey mode. Therefore, $e$-Rosita will clearly detect the angular power spectrum. On the other hand, $\mathrm{MeV}$ instruments do not have as small point spread function as X-ray instruments have. However, the Poisson term which 


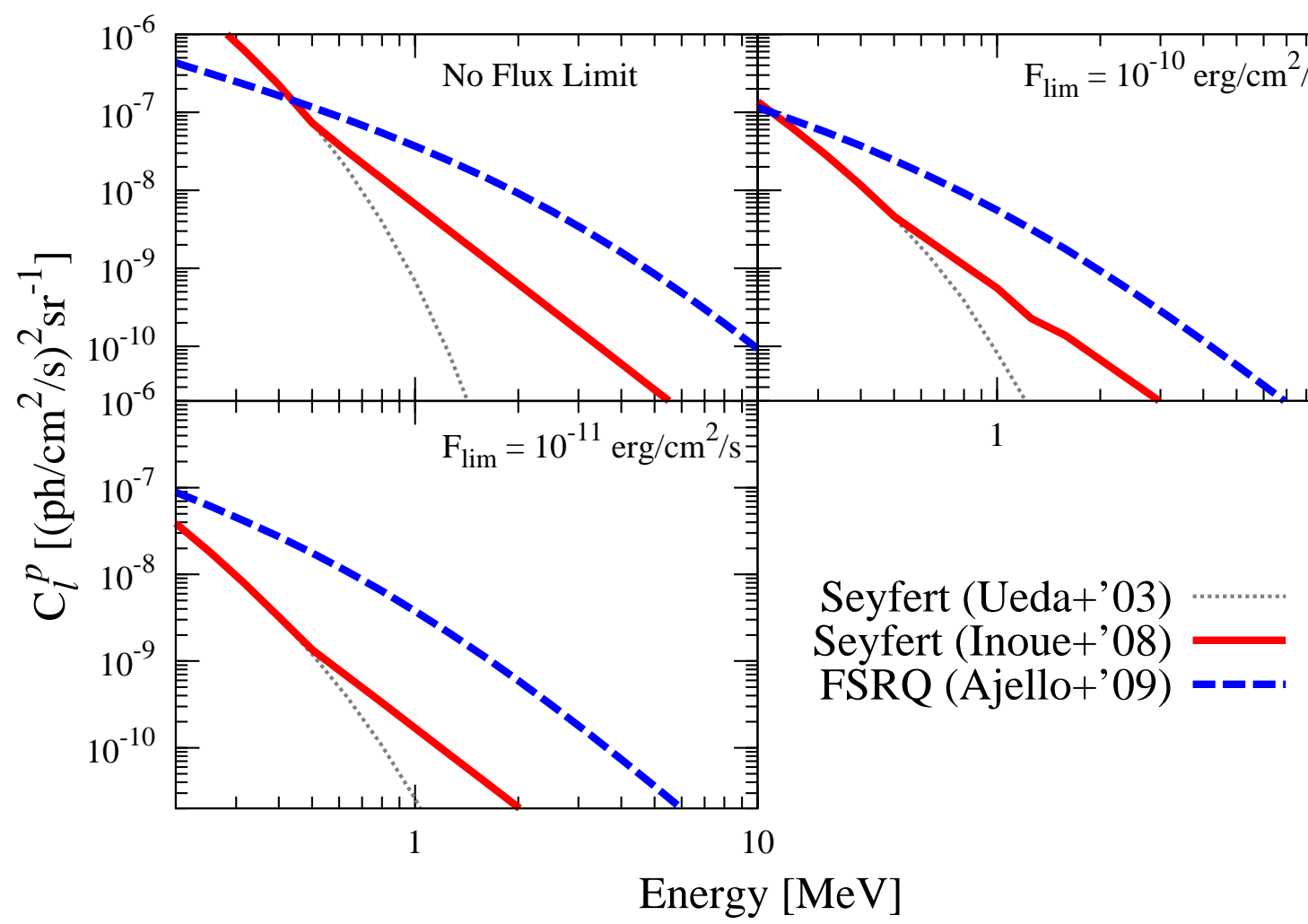

FIG. 8.- Predicted poisson term of the angular power spectrum of the cosmic MeV background at $200 \mathrm{keV}-10 \mathrm{MeV}$. Each panel shows the all sky survey case $f_{\mathrm{skv}}=1$ with the $\nu F_{\nu}$ sensitivity limit shown in the panel. Solid and dashed curve corresponds to Seyferts with non-thermal electrons in coronae (Inoue et al. 2008) and FSRQs (Aiello et al. 2009), respectively, assuming the MeV background is explained by them. For reference, we also plot the model of Seyferts with thermal cutoff (Ueda et al. 2003) by dotted curve which does not explain the MeV background radiation.

is the key to understanding the origin of the $\mathrm{MeV}$ background does not depend on the multipole.

Gravitational lensing by clusters of galaxies may change the angular power spectrum, since the lensed sources are strongly clustering and are amplified around the lensing cluster. It has been argued that the observed AGN luminosity function could be significantly affected by lensing (e.g. Turner 1980; Turner et al. 1984). The fraction of lensed AGNs at $z \lesssim 4.3$ is expected to be less than $2 \%$ at the SDSS limiting sensitivity (Wyithe \& Loeb 2002). Therefore, the lensing will not affect our results significantly.

Other populations such as galaxies are responsible for a fraction of CXB, although it is expected to be $\sim 2 \%$ (Persic \& Rephaeli 2003; Bauer et al. 2004). They may also alter the shape of the angular power spectrum. As galaxies are fainter and more numerous than AGNs, their Poisson term will be weaker than that of AGNs and their correlation term contribution arises at different multipole due to the difference of the distribution in the sky. The lensing may also alter fluc- tuation signatures of $\mathrm{CXB}$ and the $\mathrm{MeV}$ background, if they are dominated by galaxies. A deficit of surface brightness within the central regions of massive galaxy clusters, which is a strong lensing cluster, after removing detected sources has recently measured with the Herschel Space Observatory. The amplitude of the deficit is the same as the full intensity of the lensed cosmic infrared background radiation which is dominated by galaxies (Zemcov et al. 2013).

We thank Kazuo Hiroi, Megumi Shidatsu, Takeshi Tsuru, Tatsuya Sawano, Atsushi Takada, and Toru Tanimori for useful comments. YI acknowledges support by the Research Fellowship of the Japan Society for the Promotion of Science (JSPS). YI thanks the hospitality of the Center for Cosmology and AstroParticle Physics (CCAPP) at the Ohio State University. KM is supported by NASA through Hubble Fellowship grant No. 51310.01 awarded by the Space Telescope Science Institute, which is operated by the Association of Universities for Research in Astronomy, Inc., for NASA, under contract NAS 5-26555.

\section{REFERENCES}

Abdo, A. A. et al. 2010a, Physical Review Letters, 104, 101101 -. 2010b, ApJ, 720, 435

Ackermann, M. et al. 2011, ApJ, 743, 171

-. 2012a, Phys. Rev. D, 85, 083007

—. 2012b, eprint arXiv:1206.1346

Ahn, K. \& Komatsu, E. 2005a, Phys. Rev. D, 71, 021303

—. 2005b, Phys. Rev. D, 72, 061301

Ahn, K., Komatsu, E., \& Höflich, P. 2005, Phys. Rev. D, 71, 121301

Aird, J. et al. 2010, MNRAS, 401, 2531
Ajello, M., Alexander, D. M., Greiner, J., Madejski, G. M., Gehrels, N., \& Burlon, D. 2012a, ApJ, 749, 21

Ajello, M. et al. 2008, ApJ, 689, 666

-. 2009, ApJ, 699, 603

-. 2012b, ApJ, 751, 108

Akylas, A., Georgantopoulos, I., \& Plionis, M. 2000, MNRAS, 318, 1036

Alexander, D. M. et al. 2003a, AJ, 126, 539

-. 2003b, AJ, 125, 383

Ando, S. \& Komatsu, E. 2006, Phys. Rev. D, 73, 023521

-. 2013, arXiv:1301.5901 
Ando, S., Komatsu, E., Narumoto, T., \& Totani, T. 2007a, MNRAS, 376, 1635

-. 2007b, Phys. Rev. D, 75, 063519

Bandstra, M. S. et al. 2011, ApJ, 738, 8

Barcons, X., Fabian, A. C., \& Carrera, F. J. 1998, MNRAS, 293, 60

Barcons, X., Franceschini, A., de Zotti, G., Danese, L., \& Miyaji, T. 1995, ApJ, 455, 480

Basilakos, S., Georgakakis, A., Plionis, M., \& Georgantopoulos, I. 2004, ApJ, 607, L79

Basilakos, S., Plionis, M., Georgakakis, A., \& Georgantopoulos, I. 2005, MNRAS, 356, 183

Bauer, F. E. et al. 2004, AJ, 128, 2048

Baumgartner, W. H. et al. 2012, ArXiv e-prints

Blom, J. J. et al. 1995, A\&A, 298, L33

Boughn, S. \& Crittenden, R. 2004a, Nature, 427, 45

Boughn, S. P. \& Crittenden, R. G. 2004b, ApJ, 612, 647

-. 2005, MNRAS, 360, 1013

Bradshaw, E. J. et al. 2011, MNRAS, 415, 2626

Carrera, F. J., Barcons, X., Butcher, J. A., Fabian, A. C., Lahav, O., Stewart, G. C., \& Warwick, R. S. 1995, MNRAS, 275, 22

Carrera, F. J. et al. 1993, MNRAS, 260, 376

-. 2007, A\&A, 469, 27

Chen, L.-W., Fabian, A. C., Warwick, R. S., Barber, C. R., \&

Branduardi-Raymont, G. 1994, MNRAS, 266, 846

Churazov, E. et al. 2007, A\&A, 467, 529

Clayton, D. D. \& Ward, R. A. 1975, ApJ, 198, 241

Croom, S. M. et al. 2005, MNRAS, 356, 415

Cuoco, A., Komatsu, E., \& Siegal-Gaskins, J. M. 2012, Phys. Rev. D, 86, 063004

De Luca, A. \& Molendi, S. 2004, A\&A, 419, 837

de Zotti, G., Persic, M., Franceschini, A., Danese, L., Palumbo, G. G. C., Boldt, E. A., \& Marshall, F. E. 1990, ApJ, 351, 22

Dermer, C. D. \& Schlickeiser, R. 1993, ApJ, 416, 458

Di Mauro, M., Calore, F., Donato, F., Ajello, M., \& Latronico, L. 2013, arXiv: 1304.0908

Ebrero, J. et al. 2009, A\&A, 493, 55

Eisenstein, D. J. \& Hu, W. 1999, ApJ, 511, 5

Elyiv, A. et al. 2012, A\&A, 537, A131

Field, G. B. \& Rogers, R. D. 1993, ApJ, 403, 94

Fossati, G., Maraschi, L., Celotti, A., Comastri, A., \& Ghisellini, G. 1998, MNRAS, 299, 433

Fukada, Y., Hayakawa, S., Kasahara, I., Makino, F., Tanaka, Y., \& Sreekantan, B. V. 1975, Nature, 254, 398

Gandhi, P. et al. 2006, A\&A, 457, 393

Gao, Y.-T., Stecker, F. W., Gleiser, M., \& Cline, D. B. 1990, ApJ, 361, L37

Gendreau, K. C. et al. 1995, PASJ, 47, L5

Ghisellini, G., Celotti, A., Fossati, G., Maraschi, L., \& Comastri, A. 1998, MNRAS, 301, 451

Giacconi, R., Gursky, H., Paolini, F. R., \& Rossi, B. B. 1962, Physical Review Letters, 9, 439

Giacconi, R. et al. 2002, ApJS, 139, 369

Gierliński, M., Zdziarski, A. A., Poutanen, J., Coppi, P. S., Ebisawa, K., \& Johnson, W. N. 1999, MNRAS, 309, 496

Gilli, R., Comastri, A., \& Hasinger, G. 2007, A\&A, 463, 79

Greiner, J. et al. 2012, Experimental Astronomy, 34, 551

Gruber, D. E., Matteson, J. L., Peterson, L. E., \& Jung, G. V. 1999, ApJ, 520,124

Harding, J. P. \& Abazajian, K. N. 2012, J. Cosmology Astropart. Phys., 11, 26

Harrison, F. A. et al. 2013, arXiv:1301.7307

Hasinger, G., Miyaji, T., \& Schmidt, M. 2005, A\&A, 441, 417

Horiuchi, S. \& Beacom, J. F. 2010, ApJ, 723, 329

Inoue, S. \& Takahara, F. 1996, ApJ, 463, 555

Inoue, Y. 2011, ApJ, 733, 66

Inoue, Y., Inoue, S., Kobayashi, M. A. R., Makiya, R., Niino, Y., \& Totani, T. 2013, ApJ, 768, 197

Inoue, Y. \& Totani, T. 2009, ApJ, 702, 523

Inoue, Y., Totani, T., \& Ueda, Y. 2008, ApJ, 672, L5

Johnson, W. N., McNaron-Brown, K., Kurfess, J. D., Zdziarski, A. A., Magdziarz, P., \& Gehrels, N. 1997, ApJ, 482, 173

Jones, T. W., O’dell, S. L., \& Stein, W. A. 1974, ApJ, 188, 353

Katz, J. I. 1976, ApJ, 206, 910

Kinzer, R. L., Jung, G. V., Gruber, D. E., Matteson, J. L., \& Peterson, L. E. 1997, ApJ, 475, 361

Kolodzig, A., Gilfanov, M., Hütsi, G., \& Sunyaev, R. 2013, arXiv:1305.0819

Komatsu, E. et al. 2009, ApJS, 180, 330

-. 2011, ApJS, 192, 18
Koutoulidis, L., Plionis, M., Georgantopoulos, I., \& Fanidakis, N. 2013, MNRAS, 428, 1382

Kubo, H., Takahashi, T., Madejski, G., Tashiro, M., Makino, F., Inoue, S., \& Takahara, F. 1998, ApJ, 504, 693

Kushino, A., Ishisaki, Y., Morita, U., Yamasaki, N. Y., Ishida, M., Ohashi,

T., \& Ueda, Y. 2002, PASJ, 54, 327

La Franca, F. et al. 2005, ApJ, 635, 864

Lahav, O., Piran, T., \& Treyer, M. A. 1997, MNRAS, 284, 499

Lahav, O. et al. 1993, Nature, 364, 693

Lawson, K. \& Zhitnitsky, A. R. 2008, J. Cosmology Astropart. Phys., 1, 22

Li, C., Kauffmann, G., Wang, L., White, S. D. M., Heckman, T. M., \& Jing, Y. P. 2006, MNRAS, 373, 457

Lightman, A. P. \& White, T. R. 1988, ApJ, 335, 57

Lin, J. et al. 2005, ApJ, 622, 1251

Liu, B. F., Mineshige, S., \& Shibata, K. 2002, ApJ, 572, L173

Magdziarz, P. \& Zdziarski, A. A. 1995, MNRAS, 273, 837

Massaro, F. \& Ajello, M. 2011, ApJ, 729, L12

McConnell, M., Forrest, D., Ryan, J., Collmar, W., Schoenfelder, V., Steinle, H., Strong, A., van Dijk, R., Hermsen, W., \& Bennett, K. 1994, ApJ, 424, 933

Merloni, A. et al. 2012, arXiv:1209.3114

Miyaji, T., Hasinger, G., \& Schmidt, M. 2000, A\&A, 353, 25

Miyaji, T., Lahav, O., Jahoda, K., \& Boldt, E. 1994, ApJ, 434, 424

Miyaji, T. et al. 2007, ApJS, 172, 396

Mullis, C. R. et al. 2004, ApJ, 617, 192

Mushotzky, R. F., Cowie, L. L., Barger, A. J., \& Arnaud, K. A. 2000, Nature, 404, 459

Nakazawa, K. et al. 2012, in Society of Photo-Optical Instrumentation Engineers (SPIE) Conference Series, Vol. 8443, Society of Photo-Optical Instrumentation Engineers (SPIE) Conference Series

Nandra, K. \& Pounds, K. A. 1994, MNRAS, 268, 405

Narumoto, T. \& Totani, T. 2006, ApJ, 643, 81

Newsam, A. M., McHardy, I. M., Jones, L. R., \& Mason, K. O. 1999, MNRAS, 310, 255

Olive, K. A. \& Silk, J. 1985, Physical Review Letters, 55, 2362

Padovani, P., Ghisellini, G., Fabian, A. C., \& Celotti, A. 1993, MNRAS, 260, L21

Peebles, P. J. E. 1980, The large-scale structure of the universe (Princeton University Press)

Persic, M. \& Rephaeli, Y. 2003, A\&A, 399, 9

Piconcelli, E., Jimenez-Bailón, E., Guainazzi, M., Schartel, N.,

Rodríguez-Pascual, P. M., \& Santos-Lleó, M. 2005, A\&A, 432, 15

Plionis, M., Rovilos, M., Basilakos, S., Georgantopoulos, I., \& Bauer, F. 2008, ApJ, 674, L5

Porciani, C. \& Norberg, P. 2006, MNRAS, 371, 1824

Pozdniakov, L. A., Sobol, I. M., \& Siuniaev, R. A. 1977, Soviet Ast., 21, 708

Puccetti, S. et al. 2006, A\&A, 457, 501

Rasera, Y. et al. 2006, Phys. Rev. D, 73, 103518

Reeves, J. N. \& Turner, M. J. L. 2000, MNRAS, 316, 234

Revnivtsev, M., Gilfanov, M., Jahoda, K., \& Sunyaev, R. 2005, A\&A, 444, 381

Revnivtsev, M., Molkov, S., \& Sazonov, S. 2008, A\&A, 483, 425

Roche, N., Shanks, T., Georgantopoulos, I., Stewart, G. C., Boyle, B. J., \& Griffiths, R. E. 1995, MNRAS, 273, L15

Ross, N. P. et al. 2009, ApJ, 697, 1634

Sambruna, R. M. et al. 2006, ApJ, 646, 23

Sazonov, S., Revnivtsev, M., Krivonos, R., Churazov, E., \& Sunyaev, R. 2007, A\&A, 462, 57

Scharf, C. A., Jahoda, K., Treyer, M., Lahav, O., Boldt, E., \& Piran, T. 2000, ApJ, 544, 49

Schoenfelder, V. 1978, Nature, 274, 344

Schönfelder, V. et al. 2000, A\&AS, 143, 145

Seljak, U. 2000, MNRAS, 318, 203

Shen, Y. et al. 2009, ApJ, 697, 1656

Shibata, K. et al. 1995, ApJ, 451, L83

Sikora, M., Begelman, M. C., \& Rees, M. J. 1994, ApJ, 421, 153

Silverman, J. D. et al. 2008, ApJ, 679, 118

Śliwa, W., Soltan, A. M., \& Freyberg, M. J. 2001, A\&A, 380, 397

Sołtan, A., Freyberg, M. J., \& Trümper, J. 2001, A\&A, 378, 735

Soltan, A. \& Hasinger, G. 1994, A\&A, 288, 77

Sołtan, A. M., Freyberg, M., Hasinger, G., Miyaji, T., Treyer, M., \&

Trümper, J. 1999, A\&A, 349, 354

Soltan, A. M., Hasinger, G., Egger, R., Snowden, S., \& Truemper, J. 1996,

A\&A, 305, 17

-. 1997, A\&A, 320, 705 
Soltan, A. M. \& Juchniewicz, J. 1999, Astrophysical Letters and Communications, 39, 197

Sreekumar, P. et al. 1998, ApJ, 494, 523

Stevenson, S. L., Salzer, J. J., Sarajedini, V. L., \& Moran, E. C. 2002, AJ, 124,3465

Strigari, L. E., Beacom, J. F., Walker, T. P., \& Zhang, P. 2005, J. Cosmology Astropart. Phys., 4, 17

Strong, A. W., Wolfendale, A. W., \& Worrall, D. M. 1976, MNRAS, 175, 23P

Sunyaev, R. A. \& Titarchuk, L. G. 1980, A\&A, 86, 121

Tajima, H. et al. 2010, in Society of Photo-Optical Instrumentation Engineers (SPIE) Conference Series, Vol. 7732, Society of Photo-Optical Instrumentation Engineers (SPIE) Conference Series

Takada, A. et al. 2011, ApJ, 733, 13

Takahashi, T. et al. 2012, in Society of Photo-Optical Instrumentation Engineers (SPIE) Conference Series, Vol. 8443, Society of Photo-Optical Instrumentation Engineers (SPIE) Conference Series

Treister, E. \& Urry, C. M. 2005, ApJ, 630, 115

Treister, E., Urry, C. M., \& Virani, S. 2009, ApJ, 696, 110

Treyer, M. A. \& Lahav, O. 1996, MNRAS, 280, 469

Turner, E. L. 1980, ApJ, 242, L135

Turner, E. L., Ostriker, J. P., \& Gott, III, J. R. 1984, ApJ, 284, 1

Ueda, Y., Akiyama, M., Ohta, K., \& Miyaji, T. 2003, ApJ, 598, 886

Ulrich, M.-H., Maraschi, L., \& Urry, C. M. 1997, ARA\&A, 35, 445

Urry, C. M. \& Padovani, P. 1995, PASP, 107, 803
Vikhlinin, A. \& Forman, W. 1995, ApJ, 455, L109

von Ballmoos, P. et al. 2012, Experimental Astronomy, 34, 583

Watanabe, K., Hartmann, D. H., Leising, M. D., The, L., Share, G. H., \& Kinzer, R. L. 1997, in American Institute of Physics Conference Series, Vol. 410, Proceedings of the Fourth Compton Symposium, ed. C. D. Dermer, M. S. Strickman, \& J. D. Kurfess, 1223-1227

Watanabe, K., Hartmann, D. H., Leising, M. D., \& The, L.-S. 1999, ApJ, 516,285

Weidenspointner, G. et al. 2000, in American Institute of Physics

Conference Series, Vol. 510, American Institute of Physics Conference Series, ed. M. L. McConnell \& J. M. Ryan, 467-470

Worsley, M. A., Fabian, A. C., Barcons, X., Mateos, S., Hasinger, G., \& Brunner, H. 2004, MNRAS, 352, L28

Worsley, M. A. et al. 2005, MNRAS, 357, 1281

Wyithe, J. S. B. \& Loeb, A. 2002, ApJ, 581, 886

Yang, Y., Mushotzky, R. F., Barger, A. J., Cowie, L. L., Sanders, D. B., \& Steffen, A. T. 2003, ApJ, 585, L85

Yencho, B. et al. 2009, ApJ, 698, 380

Zdziarski, A. A. 1996, MNRAS, 281, L9

Zdziarski, A. A., Lubiński, P., \& Smith, D. A. 1999, MNRAS, 303, L11

Zdziarski, A. A. et al. 1994, MNRAS, 269, L55

Zemcov, M. et al. 2013, ArXiv e-prints

Zhang, P. \& Beacom, J. F. 2004, ApJ, 614, 37

APPENDIX

ANGULAR POWER SPECTRUM OF COSMIC BACKGROUND RADIATION

Following Eq. 13, the total CXB background intensity received from the direction $\hat{\boldsymbol{r}}$ can be expressed as

$$
\begin{aligned}
I(\hat{\boldsymbol{r}}, E) & =\int_{0}^{z_{\max }} d z \frac{d^{2} V}{d z d \Omega} \int_{L_{\min }}^{L\left(F_{\lim }, z\right)} d L F(L, z, E) \rho(L, z ; \hat{\boldsymbol{r}}), \\
& =\frac{c}{4 \pi} \int_{0}^{z_{\max }} d z\left|\frac{d t}{d z}\right| \int_{L_{\min }}^{L\left(F_{\lim }, z\right)} d L L(E, z) \rho(L, z ; \hat{\boldsymbol{r}}) \\
& =\frac{1}{4 \pi} \int_{0}^{r\left(z_{\max }\right)} d r \int_{L_{\min }}^{L\left(F_{\lim }, z\right)} d L L(E, z) \rho(L, z ; \hat{\boldsymbol{r}})
\end{aligned}
$$

where we assume that the distribution in $L$ is statistically independent of position and $r$ is a proper distance corresponding to a redshift $z$. The integration term for the column density $N_{H}$ is added to calculate the background flux from Seyferts. Hereinafter, we also do not show the term of $E$ and the integration range explicitly. The deviation of the CXB intensity from its mean value is

$$
\delta I(\hat{\boldsymbol{r}}) \equiv I(\hat{\boldsymbol{r}})-<I>\text {. }
$$

Following Peebles (1980), the autocorrelation function of the CXB for point sources is

$$
\begin{aligned}
C(\theta) & =<\delta I\left(\hat{\boldsymbol{r}}_{1}\right) \delta I\left(\hat{\boldsymbol{r}}_{2}\right)> \\
& =<I\left(\hat{\boldsymbol{r}}_{1}\right) I\left(\hat{\boldsymbol{r}}_{2}\right)>-<I>^{2}, \\
& =\frac{1}{16 \pi^{2}} \int d r_{1} \int d r_{2} \xi\left(\boldsymbol{r}_{1}-\boldsymbol{r}_{2}\right)\left[\int d L_{1} L_{1}\left(z_{1}\right) \rho_{X}\left(L_{1}, z_{1} ; \hat{\boldsymbol{r}}_{1}\right)\right]\left[\int d L_{2} L_{2}\left(z_{2}\right) \rho_{X}\left(L_{2}, z_{2} ; \hat{\boldsymbol{r}}_{2}\right)\right],
\end{aligned}
$$

where $\theta$ is the angle between $\hat{\boldsymbol{r}}_{1}$ and $\hat{\boldsymbol{r}}_{2}$ in units of radians and $\xi(\boldsymbol{r})$ is the two-point correlation function of AGNs, which gives the excess probability for finding a neighbor at $\boldsymbol{r}$. We set $\boldsymbol{r}_{1}=r_{1} \hat{\boldsymbol{r}}_{1}$ and $\boldsymbol{r}_{2}=r_{2} \hat{\boldsymbol{r}}_{2}$.

The Poisson term of the angular power spectrum is obtained by setting $\theta=0$ for Eq. A7 (see $\S$. 58 of Peebles 1980)

$$
C_{l}^{p}=\int d z \frac{d^{2} V}{d z d \Omega} \int d L F(L, z)^{2} \rho_{X}(L, z) .
$$

The correlation term of the angular power spectrum of the CXB is related to the correlation function by setting $\theta \neq 0$ as

$$
C_{l}^{C}=\int_{\theta \neq 0} d^{2} \theta e^{-i l \cdot \theta} C(\theta) .
$$

For the simplicity, we use small separation approximation, so-called the Limber approximation. Eq. A7becomes

$$
\begin{aligned}
C(\theta) & =\frac{1}{16 \pi^{2}} \int d r \int d u \xi(u \hat{\boldsymbol{r}}+r(z) \theta \hat{\boldsymbol{\theta}}, z)\left[\int d L L \rho_{X}(L, z)\right]^{2} \\
& =\int d z \frac{d^{2} V}{d z d \Omega} \int d u \frac{\xi(u \hat{\boldsymbol{r}}+r(z) \theta \hat{\boldsymbol{\theta}}, z)}{16 \pi^{2}(1+z)^{2} r(z)^{2}}\left[\int d L L \rho_{X}(L, z)\right]^{2}
\end{aligned}
$$


where $r$ is $\left(r_{1}+r_{2}\right) / 2, u$ is $r_{2}-r_{1}$, and we use $d r_{1} d r_{2}=d r d u$.

Then, Eq. A9 becomes

$$
\begin{aligned}
C_{l}^{C} & =\int d^{2} \theta \int d z \int d u \frac{d^{2} V}{d z d \Omega} e^{-i \boldsymbol{l} \cdot \boldsymbol{\theta}} \frac{\xi(u \hat{\boldsymbol{r}}+r(z) \theta \hat{\boldsymbol{\theta}}, z)}{16 \pi^{2}(1+z)^{2} r(z)^{2}}\left[\int d L L \rho_{X}(L, z)\right]^{2} \\
& =\int d^{2} \theta \int d z \int d u \int \frac{d^{3} k}{(2 \pi)^{3}} \frac{d^{2} V}{d z d \Omega} e^{-i \boldsymbol{l} \cdot \boldsymbol{\theta}} \frac{P_{\mathrm{AGN}}(k, z) e^{i \boldsymbol{k} \cdot(u \hat{\boldsymbol{r}}+r \theta \hat{\boldsymbol{\theta}})}}{16 \pi^{2}(1+z)^{2} r(z)^{2}}\left[\int d L L \rho_{X}(L, z)\right]^{2} \\
& =\int d^{2} \theta \int d z \int d u \int \frac{d k_{\|} d^{2} k_{\perp}}{(2 \pi)^{3}} \frac{d^{2} V}{d z d \Omega} \frac{P_{\mathrm{AGN}}(k, z) e^{-i k_{\|} \cdot u} e^{i \boldsymbol{\theta} \cdot\left(r \boldsymbol{k}_{\perp}-\boldsymbol{l}\right)}}{16 \pi^{2}(1+z)^{2} r(z)^{2}}\left[\int d L L \rho_{X}(L, z)\right]^{2} \\
& =\int d z \int d k_{\|} d^{2} k_{\perp} \frac{d^{2} V}{d z d \Omega} \frac{P_{\mathrm{AGN}}(k, z) \delta_{D}\left(k_{\|}\right) \delta_{D}^{2}\left(r \boldsymbol{k}_{\perp}-\boldsymbol{l}\right)}{16 \pi^{2}(1+z)^{2} r(z)^{2}}\left[\int d L L \rho_{X}(L, z)\right]^{2}\left[\int\right]^{2} \\
& =\int d z \frac{d^{2} V}{d z d \Omega} \frac{P_{\mathrm{AGN}}(k=l / r, z)}{16 \pi^{2}(1+z)^{2} r(z)^{4}}\left[\int d L L \rho_{X}(L, z)\right]^{2} \\
& =\int d z \frac{d^{2} V}{d z d \Omega} P_{\mathrm{AGN}}\left(k=\frac{l}{r}, z\right)\left[\int d L F(L, z) \rho_{X}(L, z)\right]^{2}
\end{aligned}
$$

where we decomposed the wave number $k$ by the components parallel and perpendicular to $\boldsymbol{r}, \boldsymbol{k}=\boldsymbol{k}_{\|}+\boldsymbol{k}_{\perp}$, and used $d^{3} k=d k_{\|} d^{2} k_{\perp}$. We also used the relation $d_{L}(z)=(1+z) r(z)$, flux-luminosity relation, and the following Fourier transformation

$$
\xi(u \hat{\boldsymbol{r}}+r(z) \theta \hat{\boldsymbol{\theta}}, z)=\int \frac{d^{3} k}{(2 \pi)^{3}} P_{\mathrm{AGN}}(k, z) e^{i \boldsymbol{k} \cdot(u \hat{\boldsymbol{r}}+r \theta \hat{\boldsymbol{\theta}})} .
$$

We also need to consider the bias of AGNs against dark matter. The power spectrum of AGNs is given by

$$
P_{\mathrm{AGN}}\left(r, z ; L_{1}, L_{2}\right)=b_{\mathrm{AGN}}\left(L_{1}, z\right) b_{\mathrm{AGN}}\left(L_{2}, z\right) P_{\text {lin }}(r, z),
$$

where $b_{\mathrm{AGN}}$ represents the clustering strength of AGNs compared with dark matter and $P_{\text {lin }}$ represents the power spectrum of linear dark matter density fluctuations. We use the linear transfer function given in Eisenstein \& Hu (1999) to calculate $P_{\text {lin }}(r)$.

Then, Eq. A17becomes

$$
C_{l}^{C}=\int d z \frac{d^{2} V}{d z d \Omega} P_{\text {lin }}\left(k=\frac{l}{r}, z\right)\left[\int d L b_{\mathrm{AGN}}(L, z) F(L, z) \rho_{X}(L, z)\right]^{2}
$$

This is the same as Eq. 16 . 